\title{
A MULTIPLE-PATCH PHASE SPACE METHOD FOR COMPUTING TRAJECTORIES ON MANIFOLDS WITH APPLICATIONS TO WAVE PROPAGATION PROBLEMS*
}

\author{
MOHAMMAD MOTAMED ${ }^{\dagger}$ AND OLOF RUNBORG M $^{\ddagger}$
}

\begin{abstract}
We present a multiple-patch phase space method for computing trajectories on two-dimensional manifolds possibly embedded in a higher-dimensional space. The dynamics of trajectories are given by systems of ordinary differential equations (ODEs). We split the manifold into multiple patches where each patch has a well-defined regular parameterization. The ODEs are formulated as escape equations, which are hyperbolic partial differential equations (PDEs) in a threedimensional phase space. The escape equations are solved in each patch, individually. The solutions of individual patches are then connected using suitable inter-patch boundary conditions. Properties for particular families of trajectories are obtained through a fast post-processing. We apply the method to two different problems: the creeping ray contribution to mono-static radar cross section computations and the multivalued travel-time of seismic waves in multi-layered media. We present numerical examples to illustrate the accuracy and efficiency of the method.
\end{abstract}

Key words. ODEs on a manifold, phase space method, escape equations, high frequency wave propagation, geodesics, creeping rays, seismic waves, travel-time

AMS subject classifications. 53C22, 65N06, 65Y20, 78A05, 78A40

\section{Introduction}

We want to compute trajectories on two-dimensional compact manifolds possibly embedded in a higher-dimensional space. The dynamics of the trajectories we consider are given by systems of ODEs in a phase space. In many problems, we need to compute a large number of trajectories. In other words, the dynamical systems of ODEs need to be integrated for many different initial conditions. Examples include geodesics computation in computational geometry [11], robotics [2] and the theory of general relativity. Our motivation for this comes from high frequency wave propagation problems. We consider the problem of scattering of a time-harmonic incident field by a bounded scatterer $D$. We split the total field into an incident and a scattered field. The scattered field in the region outside $D$ is given by the Helmholtz equation,

$$
\Delta W+n(\mathbf{x})^{2} \omega^{2} W=0, \quad \mathbf{x} \in \mathbb{R}^{3} \backslash \bar{D},
$$

where $n(\mathbf{x})$ is the index of refraction, and $\omega$ is the angular frequency. We can impose either a Dirichlet, Neumann or Robin boundary condition on the boundary of the scatterer $\partial D$ and the Sommerfeld radiation condition at infinity. The computational cost of direct numerical simulations of (1.1) grows algebraically with the frequency. Therefore, at high frequencies, numerical methods based on approximations of (1.1) are needed. Geometrical optics (GO), for example, considers simple waves,

$$
W(\mathbf{x}) \approx a(\mathbf{x}) e^{i \omega \phi(\mathbf{x})}, \quad \mathbf{x} \in \mathbb{R}^{3},
$$

when $\omega \rightarrow \infty$. The amplitude $a(\mathbf{x})$ and the phase function $\phi(\mathbf{x})$ depend only mildly on $\omega$, and the computational cost will then be independent of $\omega$. GO can be formulated either as PDEs for $\phi$ and $a$, known as eikonal and transport equations, respectively, Jin.

${ }^{*}$ Received: January 21, 2007; accepted (in revised version): June 7, 2007. Communicated by Shi

${ }^{\dagger}$ CSC, Royal Institute of Technology (KTH), 10044 Stockholm, Sweden (mohamad@nada.kth.se).

${ }^{\ddagger}$ CSC, Royal Institute of Technology (KTH), 10044 Stockholm, Sweden (olofr@nada.kth.se). 
or as a system of ordinary differential equations (ODEs). Geometrical theory of diffraction (GTD), [18] is a correction to the GO approximations by adding diffraction effects. One type of diffracted rays is creeping rays, which are generated at the shadow line of the scatterer and propagate along geodesics on the surface, continuously shedding diffracted rays in their tangential direction. A wave field, associated to a creeping ray, is generated on the surface

$$
W_{s}(\mathbf{u})=a(\mathbf{u}) e^{i \omega \phi(\mathbf{u})},
$$

where $\phi(\mathbf{u})$ and $a(\mathbf{u})$ are surface phase and amplitude and $\mathbf{u} \in \mathbb{R}^{2}$ is a parameterization of the surface. The creeping rays are related to (1.3) in the same way as the standard GO rays are related to (1.2). Similar to GO rays, creeping rays can also be formulated either as PDEs or as a system of ODEs. There are two different approaches to compute the standard GO and creeping rays and the associated wave fields in (1.2) and (1.3); Lagrangian and Eulerian methods. Lagrangian methods are based on ODEs. The simplest Lagrangian method is standard ray tracing $[6,24,13,29]$, which gives the phase and amplitude solution along a ray. Interpolation must then be applied to obtain the solution everywhere. But, in regions where rays cross or diverge this can be rather difficult. The interpolation can be simplified by using wave front methods $[38,10]$. In these methods, instead of individual rays, an interface representing a wave front is evolved. Eulerian methods, on the other hand, are based on PDEs. The PDEs are discretized on fixed computational grids to control accuracy everywhere, and there is no problem with interpolation. The simplest Eulerian methods solve the eikonal and transport equations $[37,36,8,20]$. However, these equations only give the correct solution when it is a single wave. In the case of crossing waves, more elaborate schemes have been devised based on a third formulation of geometrical optics as a kinetic equation set in phase space. A survey of this research effort, in the free-space $\mathrm{GO}$ case, is given in $[7,31,25]$. In the surface ray case, see [26, 39] for some recent works. Fomel and Sethian [9] presented a fast phase space method for computing solutions of static Hamilton-Jacobi equations in phase space. Their method is based on escape equations which are time-independent PDEs in a three-dimensional phase space. The PDE solutions, computed by a fast marching method, give the information for all trajectories from all possible starting configurations. Recently, the authors extended the fast phase space method [26] to the problem of efficiently computing all possible creeping rays on a hypersurface. The escape solutions contain information for all incident angles. The phase and amplitude of the field are then extracted by a fast post-processing. This method is computationally attractive when the solution is sought for many different sources but with the same index of refraction, for example for computing the mono-static radar cross section (RCS). The computational cost of solving the PDEs is less than the cost of tracing all rays individually. If the surface is discretized by $N^{2}$ points the complexity is $\mathcal{O}\left(N^{3} \log N\right)$, which is close to optimal. In the mono-static RCS case, direct ray tracing would cost $\mathcal{O}\left(N^{4}\right)$ if a comparable number of incidence angles $\left(N^{2}\right)$ and rays per angle $(N)$ are considered. However, it is only applicable for scatterer surfaces with simple geometries. It assumes that the surface is represented by a single parameterization, and therefore surfaces with coordinate singularities cannot be treated, and the singularity has to be excised. Most scatterer surfaces with complicated geometries, for example, cannot be represented by a single non-singular explicit parameterization. This problem can be resolved by splitting the scatterer surface into several simpler surfaces with explicit parameterizations. These multiple patches collectively cover the scatterer surface in a non-singular manner. Moreover, one can get other benefits in this way: 
1. smaller gradients in the solution by refining the patches with more highly varying velocity coefficients,

2. possibility to parallelize, since the patches can be handled independently,

3. less need for internal memory, and

4. use of the possible symmetry of the scatterer (for example, an ellipsoid).

In this paper, we consider a two-dimensional compact manifold $M$ embedded in $\mathbb{R}^{d}$ and compute trajectories on the manifold. We first consider the case when the manifold is represented by a single regular parameterization and modify the fast phase space method $[9,26]$ to a more general class of problems. Second, we consider the case when the manifold is represented by an atlas of charts and modify the singlepatch phase space method to this case. In both cases, dynamics of trajectories are given by systems of first-order ODEs. Multiple-patch (or multi-block) finite difference schemes have long been used in computational science. They are a sub-class of domain decomposition methods for solving PDEs by iteratively solving sub-problems on smaller sub-domains [5]. However, the scheme presented here is not based on iterations. Another domain decomposition method related to the multiple-patch algorithm is the slowness matching Eulerian method [34], where local single-valued solutions of the eikonal equations are patched together by slowness matching to obtain a global, multi-valued traveltime field. In Section 2, we give the governing equations describing the dynamics of trajectories on two classes of compact manifolds: the manifolds which can be represented by a single regular parameterization and the manifolds which are described by an atlas of charts. The construction of the single- and multiple-patch schemes are described in Sections 3 and 4, respectively. In Sections 5 and 6, we present applications in computing creeping rays and seismic waves, together with sample numerical results from a prototype implementation of the scheme.

\section{Governing equations}

Consider a two-dimensional compact manifold $M$ embedded in $\mathbb{R}^{d}$. We want to compute trajectories on the manifold. Since we are interested in applications to wave propagation problems, it is natural to consider the trajectories as rays, and we will use this terminology henceforth. We consider two cases: when the manifold is represented by a single regular parameterization, and when the manifold is represented by an atlas of charts. In both cases, dynamics of rays are given by systems of three first-order ODEs describing the rate of change of the rays' location and direction along the ray trajectories.

2.1. Single-patch manifolds. First, assume that the manifold can be represented by a regular parameterization $\mathbf{x}=\bar{X}(\mathbf{u})$, where $\mathbf{x} \in M$, and the parameters $\mathbf{u}=(u, v)$ belong to a set $\Omega \subset \mathbb{R}^{2}$. Note that if $M$ is a hypersurface or a plane embedded in $\mathbb{R}^{3}$, then $\mathbf{x}=(x, y, z) \in \mathbb{R}^{3}$, and if $M$ is a plane in $\mathbb{R}^{2}$, then $\mathbf{x}=(x, y) \in \mathbb{R}^{2}$. We introduce the phase space $\mathbb{P}=\mathbb{R}^{2} \times \mathbb{S}$, where $\mathbb{S}=[0,2 \pi]$, and consider the triplet $\gamma=(u, v, \theta)$ as a point in this space. Let the rays be given by a system of three ODEs

$$
\dot{\gamma}=\mathbf{g}(\gamma),
$$

where the dot denotes differentiation with respect to the parameter $\tau$ being the arc length along the rays, and $\mathbf{g}=\left(g_{1}, g_{2}, g_{3}\right)$ is a given three-vector function which is periodic in $\theta \in \mathbb{S}$. The ray trajectories on $M$ are then confined to a subdomain $\Omega_{p}=$ $\Omega \times \mathbb{S} \subset \mathbb{P}$ in phase space. Note that the parameter values $\mathbf{u}=(u, v)$ represent the rays' location $\bar{X}(\mathbf{u})$ on $M$, and the angle $\theta$ represents the direction of the rays. 
Remark 2.1. A generic Hamiltonian system with Hamiltonian $H(\mathbf{u}, \mathbf{p})$ in fourdimensional space $\Omega \times \mathbb{R}^{2}$, with $\mathbf{p} \in \mathbb{R}^{2}$, can typically be reduced to the form (2.1). Here, $\theta$ can, for instance, be an angle representing the direction of vector $\mathbf{p}$. For example, if $H=|\mathbf{p}|^{2}+V(\mathbf{u}) \equiv C$, one can reduce it by setting

$$
\mathbf{p}=(C-V(\mathbf{u}))^{1 / 2}(\cos \theta \sin \theta)^{\top},
$$

where $C$ is determined by initial data. See also Section 5 and Section 6 for more examples.

Moreover, let any information transporting along the rays, represented by a (possibly vector-valued) function $\beta(\tau)$, be given by a more general system of the form

$$
\dot{\beta}=\alpha(\gamma, \beta),
$$

where $\alpha(\gamma, \beta)$ is some given function. For example, when $\beta$ is the length of the ray, we have $\alpha \equiv 1$.

2.2. Multiple-patch manifolds. There are two main classes of problems for which representing the manifold by a single parameterization is not applicable: the manifolds which cannot be described by a single regular parameterization due to singularities, e.g. an airplane surface, and the manifolds with different (discontinuous) material properties, e.g. earth consisting of materials with different seismic velocities. The former class is of a topological and geometrical nature related to the underlying manifold, and the latter class is more related to special applications. We therefore, secondly, consider the more general case when $M$ is described by an atlas of charts $\left(M_{j}, w_{j}\right)$, with $j=1, \ldots, P$, where the sets $M_{j}$ collectively cover $M$, and the mapping $w_{j}: M_{j} \rightarrow \Omega$ is bijective. In particular, we assume that $\Omega$ is the unit square and $M_{j}$ are patches with parametric equations

$$
\mathbf{x}=\bar{X}_{j}(\mathbf{u}):[0,1]^{2} \rightarrow M_{j} \subset \mathbb{R}^{d},
$$

and the mappings are $w_{j}=\bar{X}_{j}^{-1}$. Then $M=\bigcup_{j} w_{j}^{-1}\left([0,1]^{2}\right)$. Note that although the sets are closed, we still consider $M$ as an atlas. We assume further that the patches stick together along their sides (patch boundaries) and denote the side between two connected patches $M_{j}$ and $M_{j^{\prime}}$ by $S_{j j^{\prime}}$. Note that it is possible to have $j=j^{\prime}$, for instance when $M$ is a torus. When $j \neq j^{\prime}$, we have $S_{j j^{\prime}}=M_{j} \cap M_{j^{\prime}}$. It is also possible that a patch does not share a side with another patch, for example, if the manifold has boundary (e.g. a finite cylinder). We denote such a side by $S_{0 j}$ which belongs only to $M_{j}$. Denote the set of all sides by $\mathcal{S}$. For each patch with the id number $j$, let the rays be given by a system of three equations set in $\Omega_{p}$,

$$
\dot{\gamma}=\mathbf{g}_{j}(\gamma)
$$

where $\mathbf{g}_{j}=\left(g_{1}^{j}, g_{2}^{j}, g_{3}^{j}\right)$ is a given three-vector function. Note that $\mathbf{g}_{j}$ may be different for different $j$. As before, the systems (2.3) are natural structures for Hamiltonian systems on four-dimensional spaces $\Omega \times \mathbb{R}^{2}$ with Hamiltonian $H_{j}(\mathbf{u}, \mathbf{p})$ whose order are reduced by one. Correspondingly, let any information transporting along the rays, represented by a (possibly vector-valued) function $\beta(\tau)$, be given by a system of the form

$$
\dot{\beta}=\alpha_{j}(\gamma, \beta)
$$


where $\alpha_{j}(\gamma, \beta)$ is a given function. A main difference between the numerical methods for the single patch representation of the manifold and the multiple-patch case is that in the latter we need to connect the solutions of adjacent patches and impose suitable conditions at the inter-patch boundaries. In order to treat this problem, we need to introduce a global space, which is bijective with the space $\mathbb{Z}_{P} \times \Omega_{p}$, and in which the boundary conditions are defined and can easily be handled. Here $\mathbb{Z}_{P}=\{1,2, \ldots, P\}$. We first note that by our assumptions above, there is a bijective mapping between $(j, \mathbf{u}) \in \mathbb{Z}_{P} \times \Omega$ and $\mathbf{x} \in M$, except when $\mathbf{x}$ is at patch boundaries $\left(\mathbf{x} \in S_{j j^{\prime}}\right)$. Now, let $T_{\mathbf{x}} M$ be the tangent plane (the set of tangent vectors) to $M$ at point $\mathbf{x} \in M$ and $T M=\bigcup_{\mathbf{x} \in M} T_{\mathbf{x}} M$ be the tangent bundle of $M$. The dimension of $T M$ is twice the dimension of $M$. An element of $T M$ is a pair $\Gamma:=(\mathbf{x}, \mathbf{q})$ where $\mathbf{x} \in M$ and $\mathbf{q} \in T_{\mathbf{x}} M$. We consider the unit tangent bundle $U T M$ of $M$ which contains all unit-normed tangent vectors $(\|\mathbf{q}\|=1)$. Note that $U T M$ is a three-dimensional manifold embedded in $\mathbb{R}^{2 d}$. We now want to prove that the unit tangent bundle $U T M$ is in fact the global manifold which is bijective with the space $\mathbb{Z}_{P} \times \Omega_{p}$. But, before the proof, we notice that, by construction, for each point $\Gamma=(\mathbf{x}, \mathbf{q}) \in U T M$, there is a well-defined patch id number $j=\mathcal{J}(\Gamma)$, except when $\mathbf{x}$ is on patch boundaries. We extend this function also to the patch boundaries as follows:

- if $\mathbf{x} \in S_{j j^{\prime}}$ and $\mathbf{q} \nVdash S_{j j^{\prime}}$, then $\mathcal{J}(\Gamma)=\lim _{\epsilon \rightarrow 0} \operatorname{argmin}_{j} \operatorname{dist}\left(\mathbf{x}+\epsilon \mathbf{q}, M_{j}\right)$, which means that $\mathcal{J}(\Gamma)$ is the id of the patch into which the ray starting at $\Gamma$ enters.

- if $\mathbf{x} \in S_{j j^{\prime}}$ and $\mathbf{q} \| S_{j j^{\prime}}$, then $\mathcal{J}(\Gamma)=\max \left(j, j^{\prime}\right)$.

Where by $\mathbf{q} \| S_{j j^{\prime}}$, we mean that $\mathbf{q}$ is parallel to the patch boundary in an interval around $\mathbf{x} \in S_{j j^{\prime}}$. Therefore in this case, $\Gamma$ belongs to both $U T M_{j}$ and $U T M_{j^{\prime}}$, and we can choose either of $j^{\prime}$ and $j^{\prime}$ as the value of the function $\mathcal{J}(\Gamma)$. In order to have a welldefined function, we choose the larger one. Moreover, if $\mathbf{x}$ is at a corner sharing several patches $j, j^{\prime}, j^{\prime \prime}, \ldots$, and $\mathbf{q}$ is parallel to $S_{j j^{\prime}}$, we again choose $\mathcal{J}(\Gamma)=\max \left(j, j^{\prime}\right)$. We now prove the following Lemma.

Lemma 2.2. Suppose the Jacobian $J_{j}=D_{\mathbf{u}} \bar{X}_{j} \in \mathbb{R}^{d \times 2}$ has full rank for all $(j, \mathbf{u}) \in \mathbb{Z}_{P} \times$ $\Omega$. For each $j$ there is then a bijective mapping $W_{j}: U T M_{j} \rightarrow \Omega_{p}$ given by $W_{j}(\Gamma)=\gamma$, where

$$
\Gamma=(\mathbf{x}, \mathbf{q}), \quad \mathbf{q}=\frac{J_{j}\left(w_{j}(\mathbf{x})\right) \hat{s}(\theta)}{\left|J_{j}\left(w_{j}(\mathbf{x})\right) \hat{s}(\theta)\right|}, \quad \hat{s}(\theta)=\left(\begin{array}{c}
\cos \theta \\
\sin \theta
\end{array}\right), \quad \gamma=\left(w_{j}(\mathbf{x}), \theta\right) .
$$

Moreover, there is a bijective mapping between $(j, \gamma) \in \mathbb{Z}_{P} \times \Omega_{p}$ and $\Gamma=(\mathbf{x}, \mathbf{q}) \in U T M$.

Proof. First assume that $\mathbf{x} \in M_{j}$ and $\mathbf{q} \in U T_{\mathbf{x}} M_{j}$. Since the mapping $w_{j}=\bar{X}_{j}^{-1}$ is bijective, there is $\mathbf{u}$ such that $\bar{X}_{j}(\mathbf{u})=\mathbf{x}$, given by $\mathbf{u}=w_{j}(\mathbf{x})$. Moreover, since the Jacobian $J_{j}(\mathbf{u})$ has full rank, its columns span the tangent plane at $\mathbf{x}$, and since $\mathbf{q}$ belongs to this plane, there exists a solution $\theta$ to

$$
\frac{J_{j}(\mathbf{u}) \hat{s}(\theta)}{\left|J_{j}(\mathbf{u}) \hat{s}(\theta)\right|}=\mathbf{q}, \quad \hat{s}(\theta)=\left(\begin{array}{c}
\cos \theta \\
\sin \theta
\end{array}\right) .
$$

The second statement follows since $\mathcal{J}(\Gamma)$ is well-defined for all $\Gamma \in U T M$. This proves the lemma.

Note that the atlas of charts $\left(U T M_{j}, W_{j}\right)$ describe the space $U T M=\bigcup_{j} W_{j}^{-1}\left(\Omega_{p}\right)$. Figure 2.1 shows a schematic representation of the two-dimensional manifold $M$, the three-dimensional space $U T M$ and the corresponding bijective mappings to the parameter space $\Omega$ and phase space $\Omega_{p}$. 

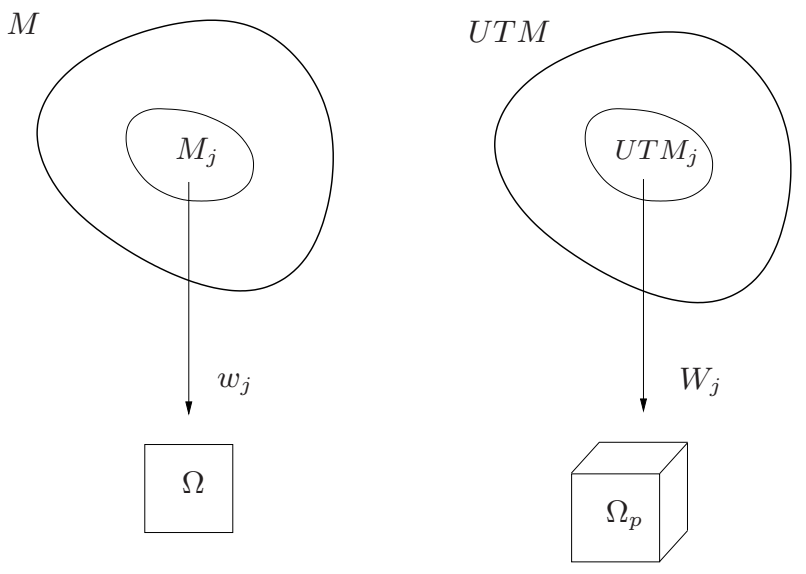

FIG. 2.1. A schematic representation of the two-dimensional manifold $M$ embedded in $\mathbb{R}^{d}$ and the three-dimensional space UTM embedded in $\mathbb{R}^{2 d}$. The bijective mappings $w_{j}$ and $W_{j}$ map a chart $j$ of these manifolds to the two-dimensional parameter space $\Omega$ and the three-dimensional phase space $\Omega_{p}$, respectively.

2.2.1. Boundary conditions. We may have different boundary conditions at the patch boundaries. In some problems, the rays are continuous at the patch boundaries. Such problems include geodesics and creeping rays computations on a hypersurface with constant index of refraction. In these problems, the boundary conditions are determined easily by the continuity of rays. In some problems, the rays may not be continuous at the patch boundaries. For example, seismic propagation in a multi-layered media with different seismic velocities is such a problem, in which the boundary conditions are determined by Snell's law of refraction or the law of reflection. As was mentioned before, the inter-patch boundary conditions are given in physical space in terms of $\Gamma \in U T M$, rather than in terms of $\gamma \in \Omega_{p}$. Let $\Gamma=(\mathbf{x}, \mathbf{q})$, where $\mathbf{x} \in S_{j j^{\prime}}$ and $j^{\prime}=\mathcal{J}(\Gamma) \neq j$, which means that the ray arrives at the side $S_{j j^{\prime}}$ from patch $M_{j}$. The inter-patch boundary condition at $S_{j j^{\prime}}$ is given by,

$$
\tilde{\Gamma}=\mathcal{L}_{j j^{\prime}}(\Gamma),
$$

where $\mathcal{L}_{j j^{\prime}}$ is some known function, and $\tilde{\Gamma}=(\tilde{\mathbf{x}}, \tilde{\mathbf{q}}) \in U T M_{\mathcal{J}(\tilde{\Gamma})}$. For example, depending on the ray arriving at the side $S_{j j^{\prime}}$ from patch $M_{j}$, we may have the following boundary conditions:

- If the ray is continuous, then $\mathcal{L}_{j j^{\prime}}$ is the identity function

$$
\tilde{\mathbf{x}}=\mathbf{x}, \quad \tilde{\mathbf{q}}=\mathbf{q} .
$$

- If the ray is refracted, then

$$
\tilde{\mathbf{x}}=\mathbf{x}, \quad \tilde{\mathbf{q}}=\tilde{\mathcal{S}}(\mathbf{x}, \mathbf{q}) .
$$

- If the ray is reflected, then

$$
\tilde{\mathbf{x}}=\mathbf{x}, \quad \tilde{\mathbf{q}}=\tilde{\mathcal{R}}(\mathbf{x}, \mathbf{q}) .
$$

Here, the functions $\tilde{\mathcal{S}}$ and $\tilde{\mathcal{R}}$ are determined by Snell's law of refraction and the law of reflection, respectively. See Section 6.2 for more details. In the next two sections, we present a patch-based phase space method for computing ray trajectories 
on manifolds. First, we consider the case when the manifold is represented by a single parameterization and construct a single-patch phase space method based on writing the systems (2.1-2.2) in an Eulerian framework. Next, we consider a wider class of manifolds which are represented by multiple parameterizations and introduce a multiple-patch phase space method based on solving the Eulerian version of systems (2.3-2.4) in each patch and connecting the solutions of individual patches using suitable inter-patch boundary conditions. In both methods, properties for particular ray families are obtained through a fast post-processing.

\section{Single-patch phase space scheme}

We consider the case when the two-dimensional manifold $M$ embedded in $\mathbb{R}^{d}$ is represented by a single regular parameterization. The objective is to compute the ray trajectories together with the information transported along them on $M$. First, the system of ODEs $(2.1-2.2)$, describing rays and other information, are formulated as time-independent Eulerian PDEs in phase space. These equations are then solved numerically on a fixed computational grid. The solution to the PDEs is post-processed to extract information for a particular family of rays.

3.1. Mathematical formulation. We consider a ray $\bar{\gamma}(\tau)$ satisfying (2.1), starting at $\bar{\gamma}(0)=\gamma=(u, v, \theta) \in \Omega_{p}$ and ending at the boundary $\partial \Omega_{p}=\partial \Omega \times \mathbb{S}$. We call this end point $(U, V, \Theta) \in \partial \Omega_{p}$ the escape point of the ray. See Figure 3.1. We then define three types of unknown escape functions for this ray, as follows:

- $F: \mathbb{P} \rightarrow \mathbb{P}, F(\gamma)=(U, V, \Theta)$ is the escape point.

- $\Phi: \mathbb{P} \rightarrow \mathbb{R}$ is the length of the ray. We also refer to this as the travel-time of the ray.

- $B: \mathbb{P} \rightarrow \mathbb{R}$ is a function representing a relation between the $\beta$-values at the escape and starting points, where $\beta$ satisfies (2.2).

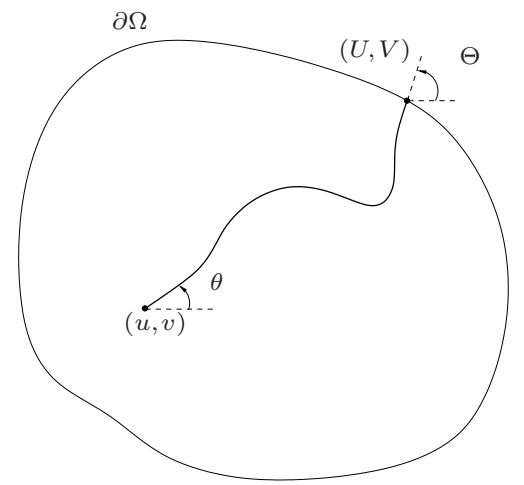

FIG. 3.1. A ray trajectory in the parameter space, starting at $\gamma=(u, v, \theta) \in \Omega_{p}$ and ending at the escape point $F(\gamma)=(U, V, \Theta) \in \partial \Omega_{p}$.

Each escape function $f(\gamma)$ of the above types satisfies an ODE,

$$
\frac{d}{d \tau} f(\gamma(\tau))=h(\gamma(\tau), f(\gamma(\tau)))
$$

where the forcing term $h$ is 0,1 and $\alpha(\gamma, f)$ for $f=F, f=\Phi$ and $f=B$, respectively. Using the chain rule, the escape PDE for each escape function $f(\gamma)$ reads

$$
g_{1}(\gamma) f_{u}+g_{2}(\gamma) f_{v}+g_{3}(\gamma) f_{\theta}=h(\gamma, f), \quad \gamma \in \Omega_{p},
$$


with the boundary condition at inflow points of $\partial \Omega_{p}$,

$$
f(\gamma)=b, \quad \gamma \in \partial \Omega_{p}^{\text {inflow }}, \quad \partial \Omega_{p}^{\text {inflow }}=\left\{\gamma \in \partial \Omega_{p} \mid \hat{n}(\gamma)^{\top} \mathbf{g}(\gamma)<0\right\},
$$

with $\hat{n}$ being the outward normal vector in the phase space. Note that for the first two types of escape functions $f=F$ and $f=\Phi$, the boundary value $b$ is $\gamma$ and 0 , respectively. For the third type $f=B$, if for instance $B$ is the difference or ratio between $\beta$-values at the escape and starting points, the boundary value are $b=0$ or $b=1$, respectively. The escape equation (3.2) is a linear hyperbolic equation, and the variable velocity coefficients $\mathbf{g}=\left(g_{1}, g_{2}, g_{3}\right)$ are known and determine the characteristic direction at every point $\gamma \in \Omega_{p}$. One important property of the solutions to the escape PDEs is that they are in general discontinuous due to discontinuous boundary conditions. This happens, for example, when a characteristic touches a boundary tangentially, such that at some points on the plane the characteristic is in-going, and suddenly it becomes out-going.

3.2. Numerical solution of the escape PDEs. We now want to solve (3.2) numerically. We discretize the phase space domain $\Omega_{p}=\Omega \times \mathbb{S}$ uniformly, setting $u_{i}=$ $i \Delta u, v_{j}=j \Delta v$ and $\theta_{k}=k \Delta \theta$, with the step sizes $\Delta u=\Delta v=\frac{1}{N}$ and $\Delta \theta=\frac{2 \pi}{N}$, assuming $\Omega$ is the unit square. Moreover, let $f_{i j k}$ approximate an escape function $f\left(u_{i}, v_{j}, \theta_{k}\right)$. In addition to the boundary condition at inflow points, since the function $f$ is periodic in $\theta$, we use periodic boundary conditions,

$$
f(u, v, 0)=f(u, v, 2 \pi)
$$

as numerical boundary conditions. There are different methods for solving the escape equation (3.2). One way is to discretize the PDEs in the phase space using a finite difference, finite volume or finite element approximation and arrive at a system of linear equations $A \bar{f}=\bar{b}$, where $A$ is an $N^{3} \times N^{3}$ matrix with a sparse structure and $\bar{b} \in \mathbb{R}^{N^{3}}$ represents the boundary conditions. This system can then be solved iteratively, and one can speed up the computations using suitable preconditioners [12, 4]. However, in the case that characteristics change direction many times in the phase space domain, it is difficult to find good preconditioners. Another way to solve the escape equations is to write them as

$$
f_{t}+g_{1} f_{u}+g_{2} f_{v}+g_{3} f_{\theta}=h
$$

and solve these time-dependent equations until the steady state $f_{t}=0$. This method can be seen as an iterative method. Finding a fast algorithm which is not much restricted by the CFL condition is analogous to finding a good preconditioner in the iterative method. Yet, another way to solve the equation (3.2) is to compute the approximate solution $f_{i j k}$ using a ray tracing method, which traces back along the characteristic to the initial boundary from each grid point $(i, j, k)$. The main drawback with this method is that it will be expensive, because one needs to trace back all $N^{3}$ points in the domain all the way to the boundary. Instead, we use a Fast Marching algorithm, given by Fomel and Sethian [9]. A similar method in two-dimensional space was also proposed in [16]. The basic idea of the algorithm is to march the solution outwards from the boundary and use the characteristic directions to update grid values. Note that in the algorithm, we always also compute $\Phi_{i j k}$ besides $f_{i j k}$. First, the grid points are divided into three classes:

- Accepted: the correct values of $f_{i j k}$ and $\Phi_{i j k}$ have been computed. 
- Considered: adjacent to Accepted for which $f_{i j k}$ and $\Phi_{i j k}$ have already been computed, but may be corrected by a later computation.

- Far: the correct values of $f_{i j k}$ and $\Phi_{i j k}$ are not known.

The major steps of the algorithm are then as follows:

0 . Start with all nodes $\left(u_{i}, v_{j}, \theta_{k}\right) \in \Omega_{p}$ in Far, and assign $\Phi_{i j k}$ at these nodes a large value. This large value needs to be greater than the length (travel-time) of every possible ray in the computational domain. Put the boundary nodes $\left(u_{i}, v_{j}, \theta_{k}\right) \in \partial \Omega_{p}^{\text {inflow }}$ in Accepted, and assign $f_{i j k}$ and $\Phi_{i j k}$ at these nodes the correct boundary values. Put each node adjacent to Accepted, for which the characteristic ${ }^{1}$ at that node points back to the boundary, in Considered. Each Considered node is then given a value by using a local cell characteristic method.

1. Take the Considered node with the smallest arrival time $\Phi_{i j k}$ as Accepted.

2. Find the octant toward which the characteristic going through that node points.

3. For each neighboring grid point in the octant which is not Accepted use the local cell characteristic method to (possibly) compute new values for $f_{i j k}$ and $\Phi_{i j k}$. In the case we can compute new values for a Far node, put it in Considered.

4. Loop to step 1 until all points are Accepted.

Since in [9] the local cell characteristic method, used in steps 0 and 3 of the algorithm, is not discussed, we will here describe a version of first and second order local cellbased ray tracing methods using a local linear and parabolic ray tracing and the Taylor expansion of the trajectory near the starting point. Consider a grid cell in $\Omega_{p}$, and assume we want to compute the value of $f_{i j k}$ at a corner of this cell, knowing the correct values of $f$ at some neighboring grid points. The output of the local ray tracing would be either a new value for $f_{i j k}$ or no new value, depending on whether the neighboring points, to which the characteristic points back, are Accepted or not. See Figure 3.2. Let $\tau$ be the arc length parameterization along the characteristic $\gamma(\tau)$. We start at $\gamma(0)=\left(u_{i}, v_{j}, \theta_{k}\right)$, where we want to compute a possibly new value, and trace backwards along the characteristic to intersect a cell face at $\gamma\left(\tau^{*}\right), \tau^{*}<0$. We Taylor expand $f$ near the starting point,

$$
f\left(\gamma\left(\tau^{*}\right)\right)=f(\gamma(0))+\tau^{*} \frac{d f}{d \tau}(\gamma(0))+\frac{\tau^{* 2}}{2} \frac{d^{2} f}{d \tau^{2}}(\gamma(0))+\mathcal{O}\left(\tau^{* 3}\right)
$$

with local truncation error $\mathcal{O}\left(\tau^{* 3}\right) \approx \mathcal{O}\left(\Delta u^{3}\right)$. Note that $\frac{d}{d \tau} f(\gamma(0))$ and $\frac{d^{2}}{d \tau^{2}} f(\gamma(0))$ in (3.3) are given by:

$$
\begin{aligned}
\frac{d}{d \tau} f(\gamma(0)) & =h(\gamma(0), f(\gamma(0))), \\
\frac{d^{2}}{d \tau^{2}} f(\gamma(0)) & =\frac{d}{d \tau} h(\gamma(0), f(\gamma(0))) \\
& =\mathbf{g}(\gamma(0)) \cdot \nabla_{\gamma} h(\gamma(0), f(\gamma(0)))+h_{f}(\gamma(0), f(\gamma(0))) h(\gamma(0), f(\gamma(0))) .
\end{aligned}
$$

Therefore, to find $f(\gamma(0))$, with accuracy of $\mathcal{O}\left(\tau^{* 3}\right)$, we need to know $\tau^{*}$ and $f\left(\gamma\left(\tau^{*}\right)\right)$. Note that for $f=F$ and $f=\Phi$, since $\frac{d}{d \tau} F(\gamma(\tau))=0$ and $\frac{d}{d \tau} \Phi(\gamma(\tau))=1$, the expansion

\footnotetext{
${ }^{1}$ We approximate the characteristic by a piecewise linear curve for a first order method and piecewise parabolic for a second order method.
} 
(3.3) reduces to

$$
\begin{gathered}
F\left(\gamma\left(\tau^{*}\right)\right)=F(\gamma(0)), \\
\Phi\left(\gamma\left(\tau^{*}\right)\right)=\Phi(\gamma(0))+\tau^{*} .
\end{gathered}
$$

3.2.1. First order method. We assume that characteristics are linear in each cell. Therefore, we can write

$$
\gamma(\tau) \approx \sigma_{1}+\sigma_{2} \tau, \quad \sigma_{1}=\gamma(0), \quad \sigma_{2}=\dot{\gamma}(0)=\mathbf{g}(\gamma(0)) .
$$

Note that $\sigma_{1}$ and $\sigma_{2}$ are known. There are six possible planes, $u=u_{i \pm 1}, v=v_{j \pm 1}$ and $\theta=\theta_{k \pm 1}$, which this line can intersect. We, therefore, get six crossing points $\tau_{1}, \ldots, \tau_{6}$, which are solutions of six linear equations. It is then clear that $\tau^{*}=\max _{\tau_{j}<0} \tau_{j}$. Knowing the crossing face and the crossing point $\gamma\left(\tau^{*}\right)$, we continue as follows:

a. If all four points of the cell face are Accepted, use these points to interpolate a value of $f\left(\gamma\left(\tau^{*}\right)\right)$. Then use the first two terms of the Taylor expansion (3.3) to compute a new value for $f_{i j k} \approx f(\gamma(0))$. Note that we need to solve a (possibly) nonlinear algebraic equation, when $h$ depends on $f$,

$$
f(\gamma(0))=f\left(\gamma\left(\tau^{*}\right)\right)-\tau^{*} h(\gamma(0), f(\gamma(0))) .
$$

Put this node in Considered. Since the method is first order, a two dimensional bilinear interpolation is used. See Figure 3.2.

b. If no points on the cell face are Accepted, do not update the value.

c. Else, continue tracing along the characteristic until either (a) or (b) occurs. Note that each time the characteristic enters a new cell, the new starting point needs to be updated.

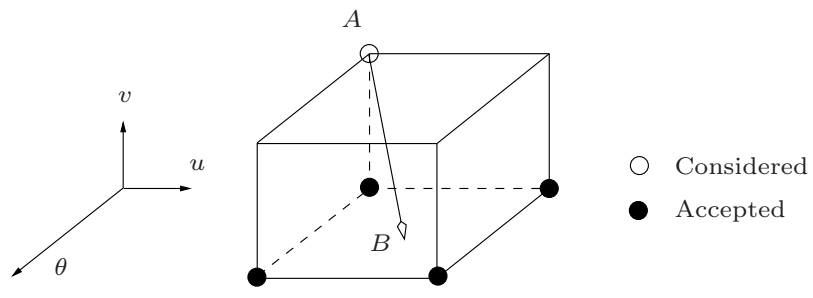

FIG. 3.2. A grid cell in $\Omega_{p}$. Point $A$ is updated by tracing the characteristic back to point $B$ and interpolating from the accepted values. Here, points $A$ and $B$ correspond to $\gamma(0)$ and $\gamma\left(\tau^{*}\right)$, respectively.

3.2.2. Second order method. We assume that characteristics are parabolic in each cell and write

$$
\gamma(\tau) \approx \sigma_{1}+\sigma_{2} \tau+\sigma_{3} \tau^{2}, \quad \sigma_{1}=\gamma(0), \quad \sigma_{2}=\dot{\gamma}(0), \quad \sigma_{3}=\frac{1}{2} \ddot{\gamma}(0)=\frac{1}{2} D_{\gamma} \dot{\gamma}(0) \dot{\gamma}(0) .
$$

Note that $\sigma_{1}, \sigma_{2}$ and $\sigma_{3}$ are known. In this case, there are nine possible cell faces which can intersect this parabola; $u=u_{i}, v=v_{j}, \theta=\theta_{k}$ and the six faces in the linear case. By intersecting the parabola with the faces, we get nine crossing points $\tau_{1}, \ldots, \tau_{9}$, which are solutions of simple quadratic equations. We then get $\tau^{*}=\max _{\tau_{j}<0} \tau_{j}$ and continue in the following way: 
a. Pick the crossing face and eight faces around it in the same plane, sharing sixteen grid points in total. If all sixteen points are Accepted, use these points to interpolate a value of $f\left(\gamma\left(\tau^{*}\right)\right)$. Then use the first three terms of the Taylor expansion (3.3) to compute a new value for $f_{i j k} \approx f(\gamma(0))$. Note that, again, we need to solve a (possibly) nonlinear algebraic equation, when $h$ depends on $f$,

$$
f(\gamma(0))=f\left(\gamma\left(\tau^{*}\right)\right)-\tau^{*} h(\gamma(0), f(\gamma(0)))-\frac{\tau^{* 2}}{2} \frac{d}{d \tau} h(\gamma(0), f(\gamma(0)))
$$

Put this node in Considered. Because the solution can be discontinuous, we use a version of two dimensional essentially non-oscillatory (ENO) interpolation based on Newton divided differences and the Newton formulation of the interpolation polynomial. Among four points in each dimension, we pick up either the left three or the right three points which have a smaller divided difference and use a second order polynomial. See [33].

b. If no points on the cell face are Accepted, do not update the value.

c. Else, continue tracing along the characteristic until either (a) or (b) occurs. Note that each time the characteristic enters a new cell, the new starting point needs to be updated.

The algorithm is a one-pass algorithm and is of complexity $\mathcal{O}\left(N^{3} \log N\right)$. Note that we use a heap sort algorithm for extracting the smallest arrival time $\Phi_{i j k}$ of Considered nodes and for inserting new updated values of Considered nodes. There is, however, no proof of convergence for the method.

3.3. Post-processing. Solutions of the escape PDEs (3.2) give the escape point, length and other information for rays with all possible starting points in the phase space. These solutions need to be post-processed to extract properties for a ray family. As an example, suppose we want to compute the length of the ray between two points $\mathbf{u}_{1}$ and $\mathbf{u}_{2}$ in the parameter space $\Omega$. We first observe that $F\left(\gamma_{1}\right)=F\left(\gamma_{2}\right)$, if and only if the points $\gamma_{1}$ and $\gamma_{2}$ lie on the same ray. We can thus find $\theta_{1}$ and $\theta_{2}$, as the solution to

$$
F\left(\mathbf{u}_{1}, \theta_{1}\right)=F\left(\mathbf{u}_{2}, \theta_{2}\right)
$$

The length is then given by $\left|\Phi\left(\mathbf{u}_{1}, \theta_{1}\right)-\Phi\left(\mathbf{u}_{2}, \theta_{2}\right)\right|$. Note that there may be multiple solutions to (3.6), giving multiple lengths. If $\mathbf{u}_{2} \in \partial \Omega$, the expression simplifies to solving

$$
\left(U\left(\mathbf{u}_{1}, \theta_{1}\right), V\left(\mathbf{u}_{1}, \theta_{1}\right)\right)=\mathbf{u}_{2},
$$

for $\theta_{1}$ to get the length $\Phi\left(\mathbf{u}_{1}, \theta_{1}\right)$. To solve (3.6), we note that since $F=(U, V, \Theta) \in \partial \Omega_{p}$ is a point on the phase space boundary, it can be reduced to a point $(S, \Theta)$ in $\mathbb{R}^{2}$, where $S$ represents the escape location on the boundary $\partial \Omega$. For example, if $\Omega=[0,1]^{2}$, we can choose $S \in[0,2 \pi]$ along $\partial \Omega$ such that $S=0, S=\pi$ and $S=2 \pi$ for $(U, V)=(0,0)$, $(U, V)=(1,1)$ and $(U, V)=(0,0)$, respectively. The left and right hand sides of (3.6) are then curves in $\mathbb{R}^{2}$ parameterized by $\theta_{1}$ and $\theta_{2}$, and solving the algebraic equation (3.6) amounts to finding crossing points of these curves. Having the discrete solutions at the points $\mathbf{u}_{1}$ and $\mathbf{u}_{2}$ for all $N$ directions, we then need to find crossing points of two complex lines of $N$ straight line segments as the solutions to (3.6). This can be done with a complexity of $\mathcal{O}(N)$; see e.g. [35]. We note that in the case that a 
second order method for solving the escape equations is used, the linear intersection algorithm will not affect second order accuracy of the method. In fact, the intersection algorithm is performed only to find the intersection's neighboring points. We use a higher order interpolation to compute the initial angles $\theta_{1}$ and $\theta_{2}$ and the escape functions corresponding to these angles. The complexity of finding the ray length between one fixed source point and all other $N^{2}$ points in $\Omega$ is then $\mathcal{O}\left(N^{3}\right)$, and the total complexity, including solving the escape PDEs, will therefore be $\mathcal{O}\left(N^{3} \log N\right)$. This is expensive for computing this so called travel-time field for only one source point. For example by using wave front tracking or solvers based on the surface eikonal equation, the complexity is $\mathcal{O}\left(N^{2}\right)$. However, if the solutions are sought for many source points, the phase space method can be more efficient. See Section 5 for such an example.

\section{Multiple-patch phase space scheme}

We now consider the more complicated and realistic case when the manifold $M$ cannot be represented by one regular parameterization. We let $M$ be described by an atlas of charts or multiple patches and want to compute the ray trajectories together with the information transported along them on the manifold. First, in each chart (patch) the ODEs of the system $(2.3-2.4)$ are formulated as time-independent Eulerian PDEs and solved numerically on a fixed computational grid in phase space. The solutions to the PDEs in each chart are then connected using suitable inter-patch boundary conditions. Information for a particular family of rays is then extracted through a fast post-processing. We describe the multiple-patch scheme and the key design choices in such a scheme, including the number and shape of patches, the treatment of inter-patch boundaries and the choice of escape boundary.

4.1. Multiple-patch construction. We first want to define a function $\mathbf{F}$ for the multiple patch case that corresponds to the single patch solution $F$ described in Section 3 . Let $\mathcal{R}$ be some curve in $M$, representing an escape boundary. We consider a ray starting at a point $\Gamma \in U T M$ and define $\mathbf{F}(\Gamma): U T M \rightarrow U T M$ as mapping the point $\Gamma$ to another point in the space $U T M$ where the projection of the ray onto $M$ first crosses $\mathcal{R}$ (assuming such a point exists). If the compact manifold has a boundary (e.g. a finite cylinder), we let this be the escape boundary, similar to the case of a single-patch manifold. Hence, $\mathcal{R}=\bigcup_{j} S_{0 j}$. However, for a compact boundaryless manifold (e.g. a sphere or a torus), there is no obvious escape boundary, as in the single patch case. In this case we will let

$$
\mathcal{R}=\bigcup_{\left(j, j^{\prime}\right) \subset R} S_{j j^{\prime}} \subset \mathcal{S}
$$

be the escape boundary, where $R$ is some index set, to be determined (see below). To compute $\mathbf{F}(\Gamma)$, we first recall that, by construction, for each point $\Gamma=(\mathbf{x}, \mathbf{q}) \in U T M$, there is a well-defined patch id number $j=\mathcal{J}(\Gamma)$ and a well-defined mapping $W_{j}$ : $U T M_{j} \rightarrow \Omega_{p}$. Now, suppose $F_{j}(\gamma)$ are the solutions to the escape PDE (3.2), with $f=F$ in $\Omega_{p}$ corresponding to each patch with $j=1, \ldots, P$. The function $\mathbf{F}(\Gamma)$ is then given recursively by

$$
\tilde{\Gamma}_{0}=\Gamma
$$

and while $\tilde{\mathbf{x}}_{n} \notin \mathcal{R}$, where $\tilde{\Gamma}_{n}=\left(\tilde{\mathbf{x}}_{n}, \tilde{\mathbf{q}}_{n}\right)$,

$$
j=\mathcal{J}\left(\tilde{\Gamma}_{n}\right), \quad \Gamma_{n+1}=W_{j}^{-1} F_{j}\left(W_{j}\left(\tilde{\Gamma}_{n}\right)\right), \quad j^{\prime}=\mathcal{J}\left(\Gamma_{n+1}\right), \quad \tilde{\Gamma}_{n+1}=\mathcal{L}_{j j^{\prime}}\left(\Gamma_{n+1}\right),
$$


where $\mathcal{L}_{j j^{\prime}}$ is the operator representing the inter-patch boundary conditions between patches $M_{j}$ and $M_{j^{\prime}}$. Then $\mathbf{F}(\Gamma)=\Gamma_{n^{*}}$, where $n^{*}$ is the smallest index for which $\mathbf{x}_{n^{*}} \in \mathcal{R}$.

REMARK 4.1. If the rays are continuous at the patch boundaries, $\mathcal{L}_{j j^{\prime}}$ will be the identity function $\left(\tilde{\Gamma}_{n+1}=\Gamma_{n+1}\right)$. From the above recursive formula, it is easy to see that, in order to compute the function $\mathbf{F}$ for all points in UTM it is enough to know the escape PDE solutions $F_{j}$ in all patches and the patch transfer functions $\mathcal{T}_{j j^{\prime}}=W_{j^{\prime}} W_{j}^{-1}$ at all sides connecting two patches $M_{j}$ and $M_{j^{\prime}}$. Note that these transfer functions can be easily calculated from the mappings $W_{j}$. As an example, in Section 5, we will discuss the computation of creeping rays which are continuous at patch boundaries. If the rays are not continuous at the patch boundaries, each time they pass a boundary, the coordinates of $\Gamma_{n+1}$ may change $\left(\tilde{\Gamma}_{n+1} \neq \Gamma_{n+1}\right)$. This happens when, for example, the rays change their direction as they enter another patch with different properties. The patch transfer functions are then changed to $\mathcal{T}_{j j^{\prime}}=$ $W_{j^{\prime}} \mathcal{L}_{j j^{\prime}} W_{j}^{-1}$. Here, transfer functions are again easily calculated from the mappings $W_{j}$ and the inter-patch boundary conditions. We will consider such examples in Section 6, where the rays change direction according to Snell's law of refraction and the law of reflection.

Similar to $\mathbf{F}(\Gamma)$, we can define the functions $\mathbf{\Phi}(\Gamma)$ and $\mathbf{B}(\Gamma)$ in $U T M$ for the multiple patch case corresponding to the single patch functions $\Phi$ and $B$ described in Section 3. Assuming $\Phi_{j}(\gamma)$ and $B_{j}(\gamma)$ are the solutions to the escape PDE (3.2), with $f=\Phi$ and $f=B$, respectively, in $\Omega_{p}$ corresponding to each patch with $j=1, \ldots, P$, we can write

$$
\boldsymbol{\Phi}(\Gamma)=\sum_{n=0}^{n^{*}-1} \Phi_{j}\left(W_{j}\left(\tilde{\Gamma}_{n}\right)\right)
$$

with $j$ and $\tilde{\Gamma}_{n}$ as in (4.2)-(4.3), and

$$
\mathbf{B}(\Gamma)=\sum_{n=0}^{n^{*}-1} B_{j}\left(W_{j}\left(\tilde{\Gamma}_{n}\right)\right)
$$

if $B$ is, for example, the difference between $\beta$-values at $\Gamma_{n^{*}}$ and $\Gamma_{0}$, and

$$
\mathbf{B}(\Gamma)=\prod_{n=0}^{n^{*}-1} B_{j}\left(W_{j}\left(\tilde{\Gamma}_{n}\right)\right)
$$

if $B$ represents, for example, the ratio between $\beta$-values.

4.2. Post-processing. Suppose we want to compute the length of a ray connecting two points $\mathbf{x}_{1} \in M_{j_{1}}$ and $\mathbf{x}_{2} \in M_{j_{2}}$. In order to find this ray, if the manifold has a boundary, we let this be the escape boundary, and the post-processing is similar to the single patch case with $F$ replaced by $\mathbf{F}$. In the case of a boundaryless manifold, we choose the boundaries of $M_{j_{1}}$ as the escape boundary $\mathcal{R}$. We then find $\mathbf{F}\left(W_{j_{1}}^{-1}\left(w_{j_{1}}\left(\mathbf{x}_{1}\right), \theta_{1}\right)\right)$ for all directions $\theta_{1} \in \mathbb{S}$. We now modify the function $\mathbf{F}(\Gamma)$ by $\mathbf{F}_{n}(\Gamma)$, where $n$ is the number of times that the ray starting at $\Gamma$ hits the escape boundary. It is therefore obvious that $\mathbf{F}_{1}(\Gamma)=\mathbf{F}(\Gamma)$. In the case where the rays at the patch boundaries are continuous, we have,

$$
\mathbf{F}_{n}(\Gamma)=\underbrace{\mathbf{F} \circ \mathbf{F} \cdots \circ \mathbf{F}}_{n \text { times }}(\Gamma) .
$$


In general, the boundary function $\mathcal{L}_{j j^{\prime}}$ must be applied in composition too. Analogously, we can define functions $\boldsymbol{\Phi}_{n}$ and $\mathbf{B}_{n}$. For all directions $\theta_{2} \in \mathbb{S}$ we then find $\mathbf{F}_{n}\left(W_{j_{2}}^{-1}\left(w_{j_{2}}\left(\mathbf{x}_{2}\right), \theta_{2}\right)\right)$. Since we do not know how many times the ray, which starts at $\mathbf{x}_{2}$ and passes through $\mathbf{x}_{1}$, hits $\mathcal{R}$, we need to find $\mathbf{F}_{n}$ for several values $n=1,2, \ldots$. See Figure 4.1 for three different cases where $n$ is 1,2 and 3 . We then find $\theta_{1}, \theta_{2}$ and

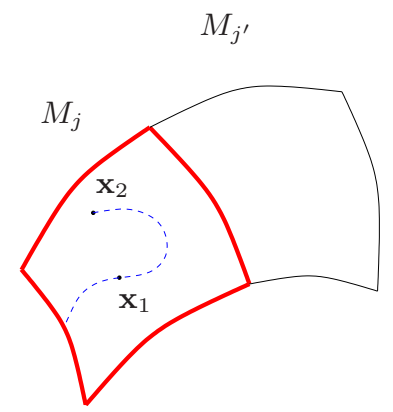

(a) $n=1,\left(j_{1}=j_{2}=j\right)$

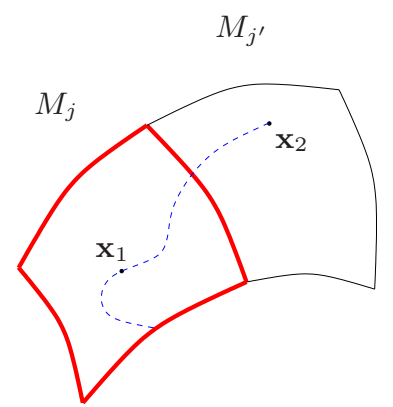

(b) $n=2,\left(j_{1}=j, j_{2}=j^{\prime}\right)$

$M_{j^{\prime}}$

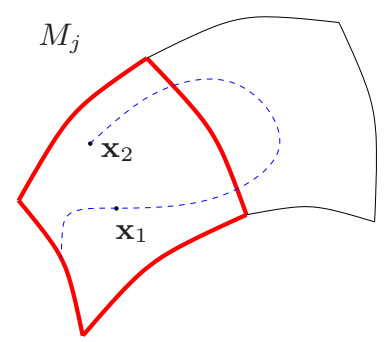

(c) $n=3,\left(j_{1}=j_{2}=j\right)$

FIG. 4.1. Two neighboring patches $M_{j}$ and $M_{j^{\prime}}$. The ray (dashed curve) starting at $\mathbf{x}_{2}$ and passing through $\mathbf{x}_{1}$, hits the escape boundary $\mathcal{R}$ (thick curves) $n$ times. Here, three different cases are shown where $n$ is 1,2 and 3 .

$n$ as the solutions to the algebraic equations

$$
\mathbf{F}\left(W_{j_{1}}^{-1}\left(w_{j_{1}}\left(\mathbf{x}_{1}\right), \theta_{1}\right)\right)=\mathbf{F}_{n}\left(W_{j_{2}}^{-1}\left(w_{j_{2}}\left(\mathbf{x}_{2}\right), \theta_{2}\right)\right),
$$

analogous to (3.6) in the single-patch case. There will be at most four systems of equations corresponding to four sides of patch $M_{j_{1}}$, for each value of $n$. The solutions to (4.5) can be computed by finding intersections of four sets of possibly crossing curves. The length is then given by

$$
\left|\boldsymbol{\Phi}\left(W_{j_{1}}^{-1}\left(\gamma_{1}\right)\right)-\boldsymbol{\Phi}_{n}\left(W_{j_{2}}^{-1}\left(\gamma_{2}\right)\right)\right|,
$$

with $\gamma_{1}=\left(w_{j_{1}}\left(\mathbf{x}_{1}\right), \theta_{1}\right)$ and $\gamma_{2}=\left(w_{j_{2}}\left(\mathbf{x}_{2}\right), \theta_{2}\right)$.

4.3. Number and shape of patches and parameterizations. One of the key design choices in such a multiple-patch scheme is the choice of patches and parameterizations. The important things are:

1. Patches should cover the physical domain with nonsingular parameterizations. 
2. Parameterizations should have small coordinate distortions to make finite differencing accurate.

3. The right hand side $h(\gamma, f)$ in the escape PDEs should be well resolved by the patch discretization.

REMARK 4.2. Using overlapping patches, one can possibly reduce the number of patches. However, the objective in this work has not been optimizing the number of patches.

4.4. Choosing escape boundary. Another key design choice is the choice of escape boundary. Two things are important about $\mathcal{R}$, and $R$ :

1. The projection of each ray of interest onto $M$ should cross $\mathcal{R}$ at some point. Otherwise $\mathbf{F}(\Gamma)$ is not well defined for all points. It is not obvious how to verify this rigorously. Having nonzero coefficients, $\mathbf{g}(\gamma) \neq 0$ everywhere is a necessary condition. However it is still possible to have rays that never reach a given boundary, see e.g. [23].

2. If the compact manifold has a boundary, we can choose this as the escape boundary, similar to the single-patch manifold.

4.5. Limitations and extra problems. There are a couple of difficulties and problems:

1. In some cases, one cannot capture all rays of interest by only one choice of escape boundary. Different choices of escape boundary might be needed. A good implementation of the algorithm will then be the one which considers different combinations of patch boundaries as the escape boundary. Note that this is done in post-processing and does not require recomputation of the $f_{j}$ solutions.

2. When a ray hits an inter-patch boundary, in order to find the escape solution at this point, we need to interpolate the discrete solutions computed on a fixed grid. The interpolation can be difficult if a ray is tangent to the interpatch boundary. One possible way to overcome such a problem is to use overlapping patches. Another possibility is to choose another atlas of charts for the manifold.

\section{Application to creeping ray computations}

A creeping rays is a type of diffracted ray which is generated at the shadow line ${ }^{2}$ of the scatterer and propagates along geodesic paths on the scatterer surface. On a perfectly conducting convex body, creeping rays attenuate along their propagation path by tangentially shedding diffracted rays and losing energy. On a concave scatterer, they propagate on the surface and importantly, in the absence of dissipation, experience no attenuation. The study of creeping rays is important in many high frequency problems, such as design of sophisticated and conformal antennas [19], antenna coupling problems [21], radar cross section (RCS) computations [3, 19, 32, 26] and control of scattering properties of metallic structures coated with dielectric materials $[28,1,22,27]$. In this section, we consider the application of the multiple-patch phase space method to computing creeping rays. Here, the computational domain is a scatterer surface which is a two-dimensional hypersurface embedded in $\mathbb{R}^{3}$. We split

\footnotetext{
${ }^{2}$ Shadow line or horizon is the locus of the points at which the incident rays are tangent to the scatterer surface.
} 
the surface into multiple patches represented by different parameterizations. The escape PDEs describing creeping rays are solved in each patch, individually. The creeping rays on the scatterer are then computed by connecting all individual solutions. The inter-patch boundaries are treated by the continuity of characteristics. We first consider the case when the scatterer surface has a regular explicit parameterization and write the governing equations for computing creeping rays. We then discuss the multiple-patch scheme and give two numerical examples where the contribution of creeping rays to mono-static RCS is computed.

5.1. Governing equations. We consider a scatterer surface with a regular explicit parameterization, represented by $\mathbf{x}=\bar{X}(\mathbf{u})$, where $\mathbf{x}=(x, y, z) \in \mathbb{R}^{3}$, and the parameters $\mathbf{u}=(u, v)$ belong to a set $\Omega \subset \mathbb{R}^{2}$. Let the scatterer be illuminated by incident rays in a direction denoted by a normalized vector $\hat{I}=\left[\imath_{1}, \imath_{2}, \imath_{3}\right]$. We assume that the shadow line $\mathbf{u}_{0}(s)$ is represented by a curve in parameter space, with $s$ being the arc-length parameterization. The objective is to compute the geodesic paths on the scatterer surface together with the phase and amplitude of the wave field of creeping rays generated on the scatterer. According to Keller and Lewis [17], the surface phase satisfies the surface eikonal equation,

$$
|\tilde{\nabla} \phi|=n,
$$

where $n(\mathbf{u})$ is the index of refraction at the surface, and $\tilde{\nabla}$ is the surface gradient, defined as

$$
\tilde{\nabla} \phi:=J G^{-1} \nabla \phi, \quad G=J^{\top} J, \quad J=\left[\bar{X}_{u} \bar{X}_{v}\right] \in \mathbb{R}^{3 \times 2} .
$$

We can write (5.1) as a Hamilton-Jacobi equation $H(\mathbf{u}, \nabla \phi)=0$, with the Hamiltonian

$$
H(\mathbf{u}, \mathbf{p}) \equiv \frac{1}{2} \mathbf{p}^{\top} G^{-1}(\mathbf{u}) \mathbf{p}-\frac{n^{2}(\mathbf{u})}{2} .
$$

Note that in the case $n=$ constant, the rays associated with the surface eikonal equation (5.1) are geodesics, or shortest paths between two points on the surface. Henceforth, we will assume $n \equiv 1$. We write (without derivation) the set of equations which are used in computing creeping rays and are obtained by reducing the order of the Hamiltonian system corresponding to (5.2) by one. For derivations see [26]. A geodesic on the surface is uniquely characterized by its location, $(u, v)$, and direction, $\theta$. Letting $\gamma:=(u, v, \theta)$, the geodesics satisfy the system of ODEs $(2.1)$ with

$$
\mathbf{g}(\gamma)=\left(\begin{array}{c}
\rho(\gamma) \cos \theta \\
\rho(\gamma) \sin \theta \\
\rho(\gamma) \mathcal{V}(\gamma)
\end{array}\right)
$$

The parameter $\tau$ is the arc length along the geodesic in the physical space, and

$$
\begin{gathered}
\rho=\rho(u, v, \theta)=\left|\bar{X}_{u} \cos \theta+\bar{X}_{v} \sin \theta\right|^{-1} \\
\mathcal{V}(\gamma)=\left(\Gamma_{11}^{1} \cos ^{2} \theta+2 \Gamma_{12}^{1} \cos \theta \sin \theta+\Gamma_{22}^{1} \sin ^{2} \theta\right) \sin \theta- \\
\left(\Gamma_{11}^{2} \cos ^{2} \theta+2 \Gamma_{12}^{2} \cos \theta \sin \theta+\Gamma_{22}^{2} \sin ^{2} \theta\right) \cos \theta
\end{gathered}
$$

where $\Gamma_{i j}^{k}(\mathbf{u})$ are Christoffel symbols. Moreover, we know that the phase $\phi$ is the length of the ray, given by (2.2) with $\beta=\phi$ and $\alpha \equiv 1$, and the amplitude $a$ is computed by,

$$
a(\tau)=a_{0} \mathcal{Q}(s, \tau)^{\frac{-1}{2}} \exp \left(-\omega^{1 / 3} \beta(\tau)\right)
$$


where $a_{0}$ is the amplitude at the starting point on the shadow line, $\mathcal{Q}(s, \tau)$ is the geometrical spreading at distance $\tau$ from the starting point, and $\beta(\tau)$ is a function representing the attenuation factor given by (2.2) with

$$
\alpha(\gamma)=\frac{q_{0}}{\rho_{g}(\gamma)} \exp \left(i \frac{\pi}{6}\left(\frac{\rho_{g}(\gamma)}{2}\right)^{1 / 3}\right), \quad q_{0} \approx 2.33811 .
$$

Here $\rho_{g}(\gamma)$ is the radius of curvature of the surface along the ray trajectory. We then let the escape function $B$ be the difference between the $\beta$-values at the escape and starting points. All escape functions $F, \Phi$ and $B$ satisfy Equ. (3.2), with the right hand side $h$ being 0,1 and $\alpha$ given by (5.5) respectively. In order to compute the amplitude, in addition to $\beta$, we need also to compute geometrical spreading. We set $\tilde{\mathbf{u}}(s, \tau):=\mathbf{u}(\tau)$, with $\tilde{\mathbf{u}}(s, 0)=\mathbf{u}_{0}(s)$ and let $\tilde{X}(s, \tau):=\bar{X}(\tilde{\mathbf{u}}(s, \tau))$ be a point on the geodesic at the distance $\tau$ from the starting point $\tilde{X}_{0}(s)=\tilde{X}(s, 0)$ on the shadow line. The geometrical spreading of the creeping ray at $\tilde{X}(s, \tau)$ in the physical space is given by, [26],

$$
\mathcal{Q}(s, \tau)=\frac{\tilde{X}_{\tau}^{\perp} \cdot \tilde{X}_{s}}{\tilde{X}_{0 \tau}^{\perp} \cdot \tilde{X}_{0 s}} .
$$

We consider a fixed shadow line $\gamma_{0}(s)=\left(u_{0}(s), v_{0}(s), \theta_{0}(s)\right)$ and define $\tilde{\gamma}(s, \tau):=\gamma(\tau)$, where $\gamma$ solves (2.1) with initial data $\gamma_{0}(s)$. Let $\mathbb{L}\left(\gamma_{0}\right)=\{\tilde{\gamma}(s, \tau): \tau \geq 0\}$ be a submanifold of phase space $\mathbb{P}$ on which the creeping rays generated at $\gamma_{0}(s)$ lie. The Eulerian version of the geometrical spreading $Q: \mathbb{L}\left(\gamma_{0}\right) \rightarrow \mathbb{R}$, restricted to $\mathbb{L}\left(\gamma_{0}\right)$ and defined as $Q(\tilde{\gamma}(s, \tau)):=\mathcal{Q}(s, \tau)$, is then given by

$$
Q(\tilde{\gamma})=\frac{[\hat{T}(\tilde{\gamma}) \times \hat{N}(\tilde{u}, \tilde{v})]^{\top} J(\tilde{u}, \tilde{v}) z}{\left[\hat{I} \times \hat{N}\left(\mathbf{u}_{\mathbf{0}}(s)\right)\right]^{\top} \tilde{X}_{0 s}(s)}, \quad \hat{T}=J \dot{\mathbf{u}},
$$

where $z=z(s, \tau)$ is a solution to

$$
D_{\gamma} F(\tilde{\gamma}) z=\frac{d}{d s} F\left(\gamma_{0}(s)\right) .
$$

For $\tilde{\gamma}$ on the boundary, i.e. $\tilde{\gamma}=F\left(\gamma_{0}\right)$, the formula (5.7) can be simplified as,

$$
Q(\tilde{\gamma})=\frac{[\hat{T}(\tilde{\gamma}) \times \hat{N}(\tilde{u}, \tilde{v})]^{\top} \hat{X}_{s}(s)}{\left[\hat{I} \times \hat{N}\left(\mathbf{u}_{\mathbf{0}}(s)\right)\right]^{\top} \tilde{X}_{0 s}}
$$

where $\hat{X}: \mathbb{R} \rightarrow \mathbb{R}^{3}$ is defined by $\hat{X}(s):=X\left(U\left(\gamma_{0}(s)\right), V\left(\gamma_{0}(s)\right)\right)$. Note that $\hat{X}_{s}(s)$ in $(5.9)$ and $D_{\gamma} F(\tilde{\gamma})$ and $F_{s}\left(\gamma_{0}(s)\right)$ in (5.8) can be computed by numerically differentiating the solution to the PDEs in (3.2) with $f=F$, as was done in [26]. Instead, one can also directly compute $\tilde{X}_{s}$ in (5.6) by adding other ODEs to the geodesic system (2.1) as follows: First, we note that $\tilde{X}_{s}=J \tilde{\mathbf{u}}_{s}$. We then differentiate (2.1) with respect to $s$ and derive the following ODE system

$$
\dot{\tilde{\gamma}}_{s}=D_{\gamma} \mathbf{g} \tilde{\gamma}_{s}, \quad \tilde{\gamma}_{s}(s, 0)=\tilde{\gamma}_{0 s}(s) .
$$

By solving this ODE, $\tilde{\mathbf{u}}_{s}$, and therefore $\tilde{X}_{s}$, can be computed. One can also write the escape PDE for (5.10) in the same way as before and post-process the phase space solution. 
5.2. Multiple-patch scheme. We now split the scatterer surface $M$ into several simpler surfaces with explicit regular parameterizations. As before, let $M$ be given by an atlas of charts $\left(M_{j}, w_{j}\right)$, where the patches $M_{j} \subset \mathbb{R}^{3}$ have the parametric equations $\mathbf{x}=\bar{X}_{j}(\mathbf{u}):[0,1]^{2} \rightarrow M_{j}$ and collectively cover $M$. Moreover, the mappings $w_{j}=\bar{X}_{j}^{-1}: M_{j} \rightarrow[0,1]^{2}$ are bijective. Since on a geodesic $\hat{T}$ in (5.7) has unit length, we can consider the unit tangent bundle $U T M$ of $M$ as the global space. Note that UTM is a three-dimensional manifold embedded in $\mathbb{R}^{6}$. By Lemma 2.2 , there is therefore a bijective mapping $W_{j}: U T M_{j} \rightarrow \Omega_{p}$ for each $j$, defined by $W_{j}(\Gamma)=\gamma$, with $\gamma$ and $\Gamma$ as in (2.5). Knowing the bijective mappings $w_{j}$ and $W_{j}$, and the solution to the escape PDEs in each patch, $F_{j}, \Phi_{j}$ and $B_{j}$, we can compute the multiple-patch escape functions $\mathbf{F}, \boldsymbol{\Phi}$ and $\mathbf{B}$ as described in Section 4.1.

5.3. Post-processing. In order to compute phase and amplitude of a ray family, post-processing of the solutions to the escape PDEs (3.2) is needed. For a given illumination direction, assume that the shadow line is known and given by $\Gamma_{0}(s)$ in the unit tangent bundle $U T M$. For each point $\mathbf{x} \in M_{j}$ covered by the surface wave, there is at least one creeping ray which starts at the shadow line and passes through that point. In order to find this ray, assuming the scatterer surface is boundaryless, we first choose the escape boundary $\mathcal{R}$ as the boundaries of $M_{j}$. Note that in the case of a surface with boundary, we choose its boundary as the escape boundary, and the post-processing will be similar to the single-patch case discussed in [26]. We then find $\mathbf{F}\left(W_{j}^{-1}\left(w_{j}(\mathbf{x}), \theta\right)\right)$ for all directions $\theta \in \mathbb{S}$. Moreover, for all points on the shadow line we find $\mathbf{F}_{n}\left(\Gamma_{0}(s)\right)$, defined by (4.4), with $n=1,2, \ldots$. We then find $s=s^{*}, \theta=\theta^{*}$ and $n=n^{*}$ as the solutions to the algebraic equations

$$
\mathbf{F}\left(W_{j}^{-1}\left(w_{j}(\mathbf{x}), \theta\right)\right)=\mathbf{F}_{n}\left(\Gamma_{0}(s)\right),
$$

analogous to (3.6) in the single-patch case. There will be at most four systems of equations corresponding to four sides of patch $M_{j}$, for each value of $n$. The solutions to (5.11) can be computed by finding intersections of four sets of possibly crossing curves. Now we can use (5.8) to compute $z$ with $\gamma_{0}=W_{j_{0}}\left(\Gamma_{0}\left(s^{*}\right)\right)$ and $\tilde{\gamma}=\left(w_{j}(\mathbf{x}), \theta^{*}\right)$, where $j_{0}=\mathcal{J}\left(\Gamma_{0}\left(s^{*}\right)\right)$. Note that $F(\tilde{\gamma})$ and $F\left(\gamma_{0}\right)$ in the left and right hand sides of (5.8) are replaced by $W_{j}\left(\mathbf{F}\left(W_{j}^{-1}(\tilde{\gamma})\right)\right)$ and $W_{j}\left(\mathbf{F}_{n^{*}}\left(\Gamma_{0}\left(s^{*}\right)\right)\right)$, respectively. The geometrical spreading $Q(\tilde{\gamma})$ at point $\mathbf{x}$ will be therefore computed by (5.7), and phase and amplitude are given by

$$
\begin{gathered}
\phi\left(w_{j}(\mathbf{x})\right)=\phi_{0}+\boldsymbol{\Phi}\left(W_{j_{0}}^{-1}\left(\gamma_{0}\right)\right)-\mathbf{\Phi}\left(W_{j}^{-1}(\tilde{\gamma})\right), \\
A(\tilde{\gamma})=A_{0} Q(\tilde{\gamma})^{\frac{-1}{2}} \exp \left(-\omega^{\frac{1}{3}}\left(\mathbf{B}\left(W_{j_{0}}^{-1}\left(\gamma_{0}\right)\right)-\mathbf{B}\left(W_{j}^{-1}(\tilde{\gamma})\right)\right)\right),
\end{gathered}
$$

where $\phi_{0}$ and $A_{0}$ are the phase and amplitude at the point $\gamma_{0}$, respectively.

5.4. Example 1: a scalene ellipsoid. We consider the scatterer surfaces to be a scalene ellipsoid (an ellipsoid with different semi-axes) and apply the multiplepatch phase space method to compute the contribution of backscattered creeping rays to mono-static RCS, i.e., the rays that propagate on the surface of the scatterer and return in the opposite direction of incident waves. We assume that the incoming amplitudes are one at attachment points on the shadow line and compute the backscattered amplitude at detachment points on the shadow line. We also compute 
the length of the backscattered rays. We consider an ellipsoid given by

$$
\frac{x^{2}}{a^{2}}+\frac{y^{2}}{b^{2}}+\frac{z^{2}}{c^{2}}=1,
$$

with $a=2, b=1$ and $c=0.5$. Since there is no single non-singular parameterization for the ellipsoid, we split it into six patches with non-singular parameterizations (see Figure 5.1) and solve for $f(\gamma)$ in each patch, as described in Section 3.2. In order

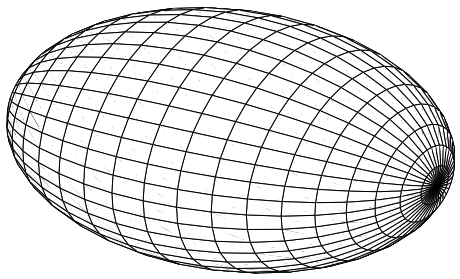

(a)

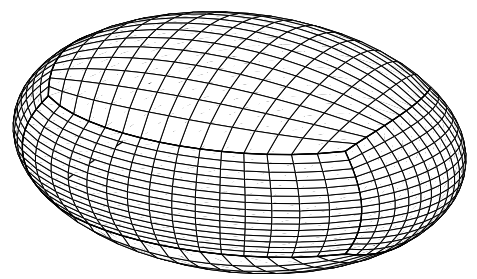

(b)

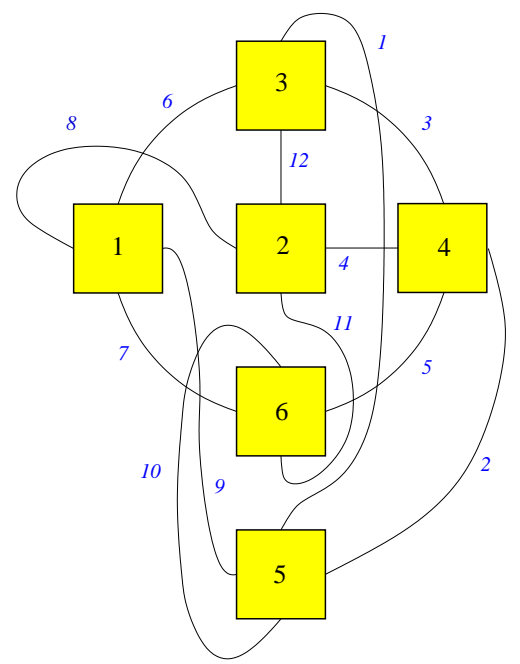

(c)

FIG. 5.1. The upper left figure shows an ellipsoid with a single patch parameterization which is singular at two poles. The upper right figure shows the ellipsoid divided into 6 patches. Note that the singularities have been removed using non-singular multiple parameterizations. The lower figure shows the structure of patches and patch boundaries in parameter space. Patches $j=1, \ldots, 6$ correspond to left, front, up, right, back and down patches, respectively. These 6 patches share 12 sides in total, shown with italic numbers.

to find the backscattered creeping ray by post-processing, we first choose the escape boundary consisting of six sides, as highlighted in Figure 5.2. We then continue as follows:

0. Given a pair of incident angles $\left(\Psi_{1}, \Psi_{2}\right) \in\left[0,90^{\circ}\right]^{2}$, find the incident direction $\hat{I}=\left[\sin \Psi_{1} \cos \Psi_{2}, \cos \Psi_{1} \cos \Psi_{2}, \sin \Psi_{2}\right]$. 


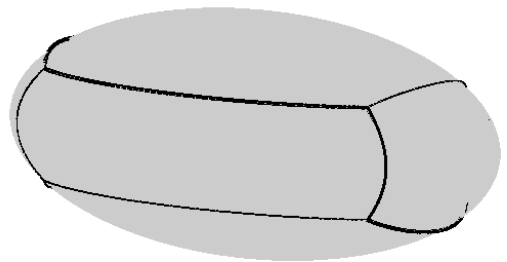

FIG. 5.2. The ellipsoid with its patch boundaries. Thick lines show the escape boundary.

1. Find the shadow line $\gamma_{0}(s)=\left(\mathbf{u}_{0}(s), \theta_{0}(s)\right)$ in the phase space $\Omega_{p}$ using the relations $\hat{N}^{\top} \hat{I}=0$ and $\hat{T}\left(\gamma_{0}(s)\right)=\hat{I}$ in patch $j(s)$. Let the parameterization of the shadow line be discretized in $N$ grid points $\left\{s_{n}\right\}$ with $n=1, \ldots, N$.

2. For each point on the shadow line find $\mathbf{F}\left(W_{j\left(s_{n}\right)}^{-1}\left(\gamma_{0}\left(s_{n}\right)\right)\right)$ as discussed in Section 4.1.

3. A backscattered ray starting at attachment point $s_{a}$ and ending at detachment point $s_{d}$ on the shadow line should satisfy

$$
\mathbf{F}\left(W_{j_{a}}^{-1}\left(\gamma_{0}\left(s_{a}\right)\right)\right)=\mathbf{F}\left(W_{j_{d}}^{-1}\left(\gamma_{0}\left(s_{d}\right)\right)\right)+C,
$$

where $j_{a}=j\left(s_{a}\right)$ and $j_{d}=j\left(s_{d}\right)$, and $C$ is a constant accounting for the fact that the directions of creeping rays starting at $s_{a}$ and $s_{d}$ differ by $\pi$ on the escape boundary. The right and left hand sides of this equation can be represented as six sets of curves in $\mathbb{R}^{2}$ parameterized by $s$, corresponding to six sides of the escape boundary. To find the backscattered ray we need to find crossing points of these curves, as is done in the single-patch case.

4. For each crossing point, there is a pair of backscattered rays (two backscattered rays lying on top of each other); one starting at point $s_{a}$ and ending at point $s_{d}$, the other starting at point $s_{d}$ and ending at point $s_{a}$. Although these two rays have the same lengths, they do not have the same geometrical spreading and therefore not the same amplitude. Compute two geometrical spreadings as described in Section 5.3 with $\gamma_{0}=\gamma\left(s_{d}\right)$ and $\tilde{\gamma}=\gamma\left(s_{a}\right)$ for the first backscattered ray and $\gamma_{0}=\gamma\left(s_{a}\right)$ and $\tilde{\gamma}=\gamma\left(s_{d}\right)$ for the second one.

5. The length and amplitudes are then computed as,

$$
\begin{gathered}
\phi=\boldsymbol{\Phi}\left(\Gamma_{s_{a}}\right)+\boldsymbol{\Phi}\left(\Gamma_{s_{d}}\right), \quad \Gamma_{s_{a}}=W_{j_{a}}^{-1}\left(\gamma\left(s_{a}\right)\right), \quad \Gamma_{s_{d}}=W_{j_{d}}^{-1}\left(\gamma\left(s_{d}\right)\right), \\
A_{1}=Q\left(\gamma\left(s_{a}\right)\right)^{\frac{-1}{2}} \exp \left(-\omega^{\frac{1}{3}}\left(\mathbf{B}\left(\Gamma_{s_{a}}\right)+\mathbf{B}\left(\Gamma_{s_{d}}\right)\right)\right), \\
A_{2}=Q\left(\gamma\left(s_{d}\right)\right)^{\frac{-1}{2}} \exp \left(-\omega^{\frac{1}{3}}\left(\mathbf{B}\left(\Gamma_{s_{a}}\right)+\mathbf{B}\left(\Gamma_{s_{d}}\right)\right)\right) .
\end{gathered}
$$

Figure 5.3 shows the backscattered rays for two different incident angles. There are three pairs of backscattered rays which can be detected by the algorithm. Every two 

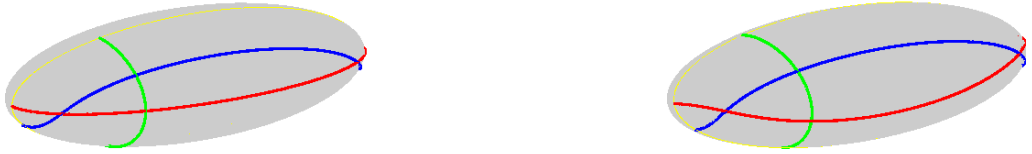

FIG. 5.3. The left figure shows the backscattered creeping rays (thick curves) for $\Psi_{1}=30$ and $\Psi_{2}=0$. The right figure shows the backscattered creeping rays for $\Psi_{1}=30$ and $\Psi_{2}=10$. Thin curves represent the shadow lines.

rays of each pair lie on top of each other. We note that in [26], because of using a single patch and excising the singularity at two poles, only the shortest backscattered ray could be captured. Figure 5.4 shows the length and amplitudes of the shortest backscattered ray for different incident angles, with $\omega=1$. The peaks in the amplitude correspond to caustic backscattered creeping rays which have infinite amplitudes. Such rays are particularly important in near-field RCS computations. However, in far-field RCS, due to the the geometrical spreading outside the scatterer, their contribution may not be as important. Figure 5.5 shows the convergence of length and amplitudes of the backscattered creeping ray for a fixed vertical angle $\Psi_{2}=70$ and different horizontal incident angles $\Psi_{1} \in\left[-90^{\circ}, 90^{\circ}\right]$. We use a second-order Fast Marching algorithm on a coarse grid of the size $50^{3}$ and a fine grid of the size $100^{3}$. We compare them with a reference solution obtained by a high-order ray-tracing method. The rate of convergence confirms the second-order accuracy of the algorithm. We note that compared to the results in [26], where a first-order algorithm was used, the accuracy of the amplitude has been improved dramatically. This shows that using a first-order-accurate method for computing the phase and amplitude results in a worse relative error for the amplitude than for the phase. Therefore, higher-order algorithms are required to obtain low relative errors for the amplitude, as observed also in [30]. The complexity of using the fast phase space method proposed here consists of two parts. First, the cost of solving the PDEs by the Fast Marching method is $\mathcal{O}\left(N^{3} \log N\right)$. Second, the cost of finding the backscattered rays for each shadow line is $\mathcal{O}(N)$. For all $N^{2}$ shadow lines, the cost is $\mathcal{O}\left(N^{3}\right)$. Therefore the total complexity will be $\mathcal{O}\left(N^{3} \log N\right)$. The total cost of other methods, such as wave-front tracking and solvers based on the surface eikonal equation, will be $\mathcal{O}\left(N^{4}\right)$, if the cost for each shadow line is $\mathcal{O}\left(N^{2}\right)$. In this case, using the phase-space method will then be much faster.

REMARK 5.1. A graph structure can be useful for a general computer implementation. The topology of the surface can be described by a graph, in which each patch is a node and the edges go between connected patches. Figure 5.6 a shows the graph corresponding to the ellipsoid divided into six patches which are connected through twelve sides (see Figure 5.1). The graph therefore has six nodes and twelve edges. We can also introduce another topology graph, in which the nodes are the sides of 

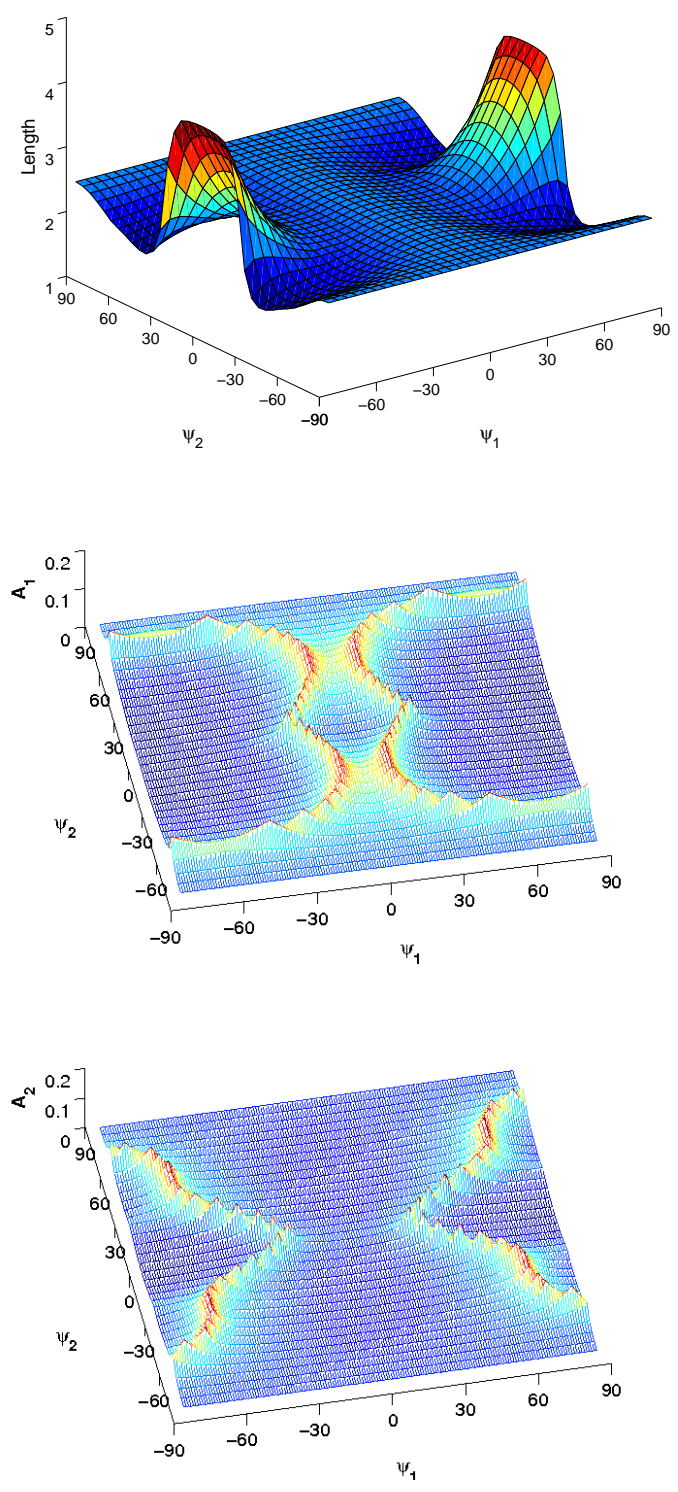

FIG. 5.4. Length and amplitudes (with $\omega=1$ ) of backscattered creeping rays for many illumination angles.

the patches and the edges correspond to the patches themselves. Each node (side) is therefore connected to six other nodes through two patches which are connected by that side. See Figure 5.6b. This structure can be useful for imposing inter-patch boundary conditions and computing $\mathbf{F}$. 

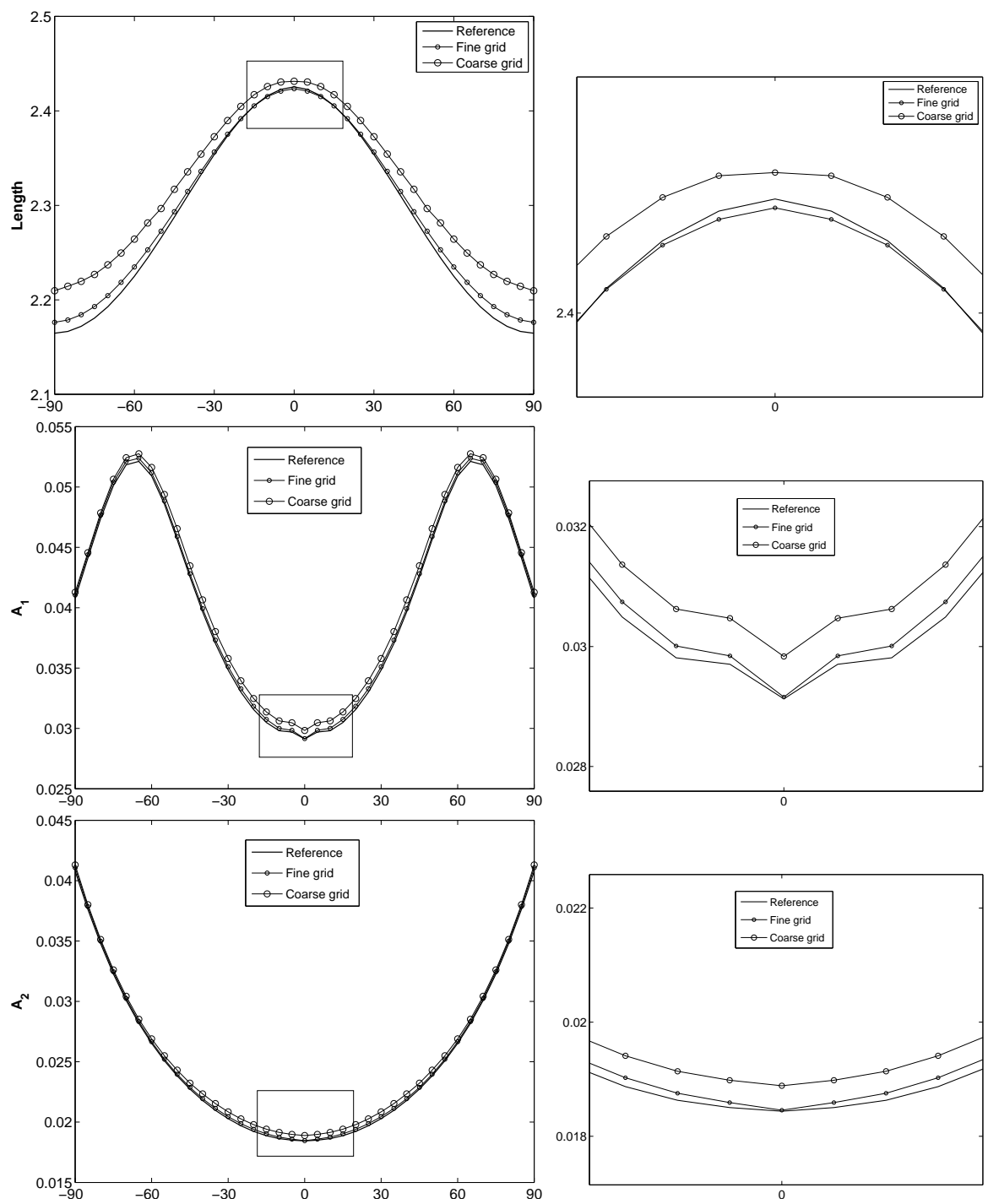

FIG. 5.5. Length and amplitude (with $\omega=1$ ) of the backscattered creeping rays for different horizontal incident angles and a fixed vertical angle $\Psi_{2}=70^{\circ}$. By refining the grid, solutions of the second order phase-space algorithm converge to a reference solution obtained by a high order ray tracing method with a correct rate. The right figures show zoomed views of left figures.

5.5. Example 2: a balloon. We consider a balloon-shaped surface consisting of a hemisphere in the positive side of the $z$-axis, centered at the origin and with radius $r$, and the surface created by rotating the part of the parabola $z^{2}=2 r(r-y)$ over the interval $-\sqrt{2} r \leq 0$ about the $z$-axis. This is a simple smooth version of the conehemisphere studied in [3] as a model for low-observable objects where creeping rays are important for RCS. We divide this surface into six patches, as shown in Figure 5.7; The hemisphere is split into five patches $j=1, \ldots, 5$, and the parabolic part is represented by one patch $j=6$. We excise the singularity at the vertex of the balloon by cutting it off. The lower boundary of patch $j=6$ will therefore be an excision boundary and is 


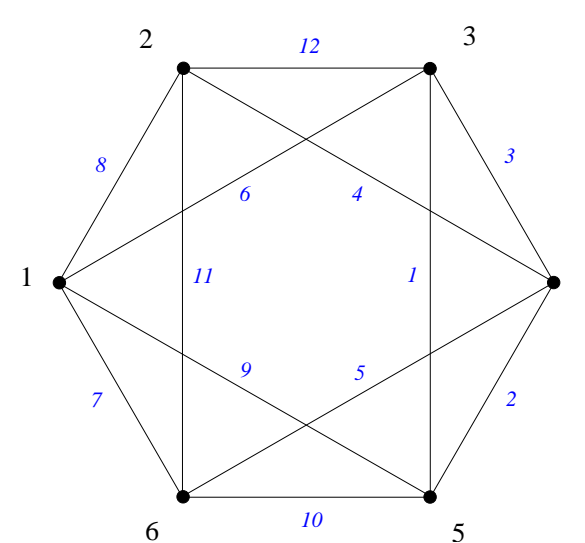

(a)

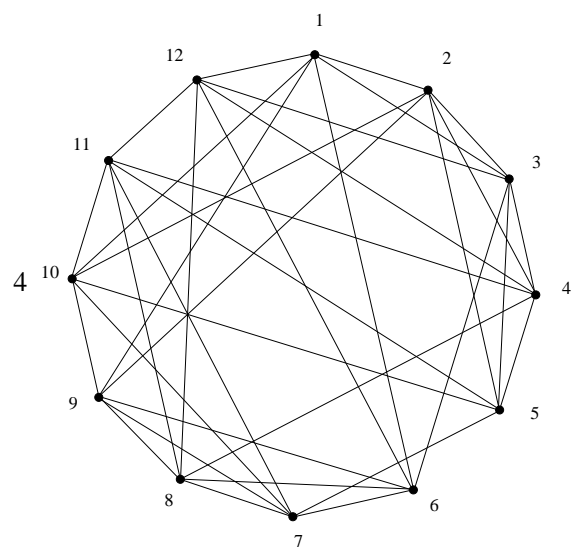

(b)

FIG. 5.6. Representation of an ellipsoid divided into 6 patches by two different graph structures. The left figure shows the graph with 6 nodes and 12 edges. Here, the nodes 1 to 6 denotes the left, front, up, right, back and down patches, respectively. The right figure shows the graph with 12 nodes and 72 edges.

not considered as a patch boundary. We also partition the upper boundary of patch $j=6$ into four boundaries connecting to lower boundaries of patches $j=1, \ldots, 4$. Note that the left and right boundaries of patch $j=6$ are in fact the same. Therefore, there are in total thirteen sides connecting six patches. See Figure 5.7. Since the

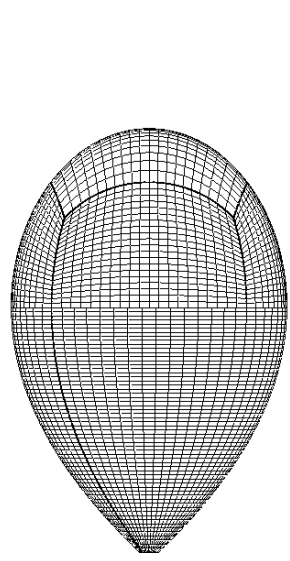

(a)

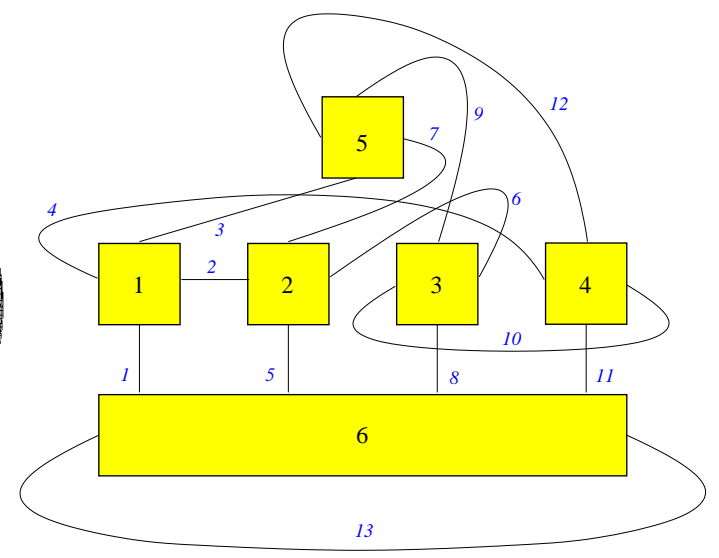

(b)

FIG. 5.7. The left figure shows the balloon divided into 6 patches. The right figure shows the structure of patches and patch boundaries in parameter space. Patches $j=1, \ldots, 6$ correspond to front, right, back, left, up and down patches, respectively. These 6 patches share 13 sides in total, shown with italic numbers.

surface is symmetric about the $z$-axis, we consider a fixed horizontal incident angle $\Psi_{1}=90^{\circ}$, and due to symmetry about the $y z$-plane, we consider the vertical angles $\Psi_{2} \in\left[-90^{\circ}, 90^{\circ}\right]$. Figure 5.8 shows the backscattered rays for two different incident angles $\Psi_{2}=40^{\circ}$ and $\Psi_{2}=-40^{\circ}$. For positive vertical-incident angles, there are four 
pairs of backscattered rays which can be detected by the algorithm. Two of them are symmetric and have the same length and amplitudes. For negative vertical-incident angles, only one backscattered ray can be captured. We notice that in the case $\Psi_{2}=$ $90^{\circ}$, there will be infinitely many backscattered rays which results in high observability of the object in this incident direction. On the other hand, for $\Psi_{2}=-90^{\circ}$, there will be no backscattered ray because we have excised the vertex. In fact, even if we did not excise it, all creeping rays would go to the vertex and diffract in different directions. Figure 5.9 shows the length and amplitude of backscattered rays in a polar coordinate
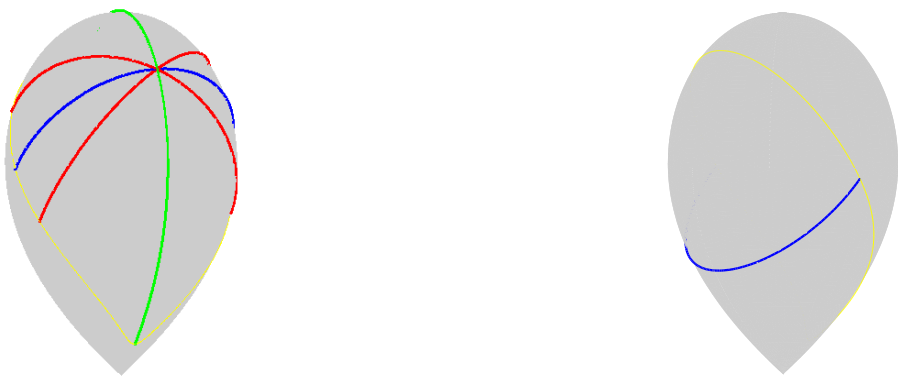

FIG. 5.8. Backscattered creeping rays (thick curves) for $\Psi_{2}=40^{\circ}$ (left figure) and $\Psi_{2}=-40^{\circ}$ (right figure). Thin curves represent the shadow lines.

system for all incident directions $\Psi \in\left[0^{\circ}, 360^{\circ}\right]$. The angles $\Psi \in\left[0^{\circ}, 90^{\circ}\right]$ in the polar system correspond to $\Psi_{2} \in\left[0^{\circ},-90^{\circ}\right]$, and the angles $\Psi \in\left[270^{\circ}, 360^{\circ}\right]$ correspond to $\Psi_{2} \in\left[90^{\circ}, 0^{\circ}\right]$. The values for $\Psi \in\left[90^{\circ}, 270^{\circ}\right]$ are then calculated using the symmetry of the surface about the $y z$-plane.

\section{Application to seismic wave computations}

The inhomogeneity of earth causes deflection and reflection of seismic waves. The numerical study of seismic wave propagations, therefore, helps us to learn about the inhomogeneous structure of earth, which is important in direct and inverse problems of seismology and seismic exploration of oil. In this section, we apply the multiplepatch phase space method to compute the travel-times of seismic rays. We consider a two-dimensional multi-layered medium whose different layers have different wave speeds. We split the medium into multiple patches corresponding to different layers. The escape PDEs describing seismic waves are solved in each patch, individually. The travel-times of the waves in the medium are then computed by connecting all individual solutions. The inter-patch boundaries are treated by Snell's law and the law of reflection. We first consider the case when the medium has a regular explicit parameterization and derive the governing equations. We then discuss the multiplepatch scheme and give a numerical example for computing the travel-times.

6.1. Governing equations. Consider a two-dimensional medium $M$ represented by parametric equations $\mathbf{x}=\bar{X}(\mathbf{u})$, where $\mathbf{x}=(x, y) \in M \subset \mathbb{R}^{2}$ and $\mathbf{u}=(u, v) \in$ $\Omega \subset \mathbb{R}^{2}$. The phase $\phi$ of the wave satisfies the eikonal equation,

$$
|\nabla \phi|=n(\mathbf{x}),
$$



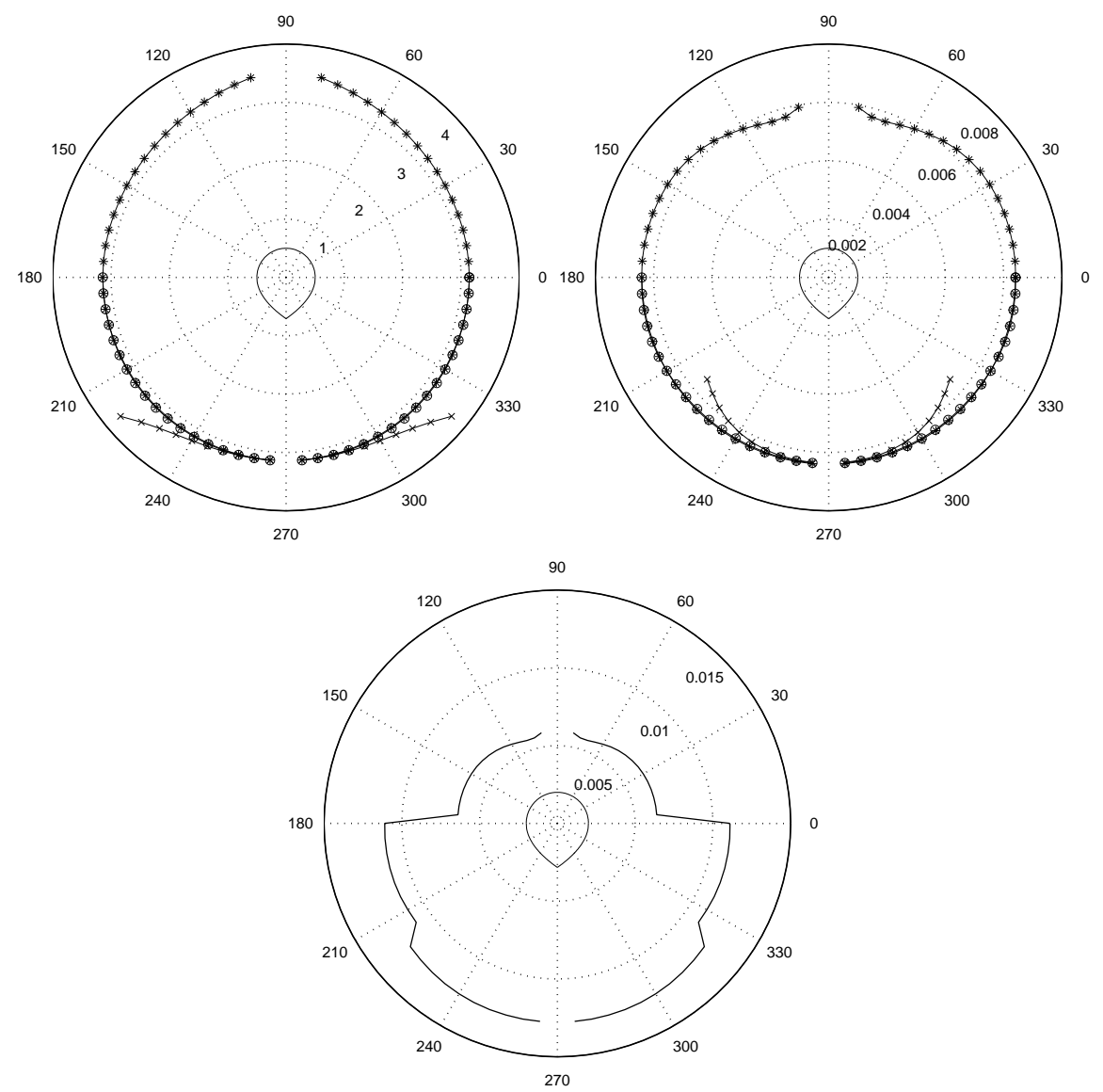

FIG. 5.9. Length and amplitude (with $\omega=1$ ) of the backscattered creeping rays for all illumination directions $\Psi \in\left[0^{\circ}, 360^{\circ}\right]$. The upper left and right figures show the length and amplitude of the backscattered rays, respectively. There are four pairs of rays among which two (illustrated by o) are symmetric. Note that at $\Psi=90^{\circ}\left(\Psi_{2}=-90^{\circ}\right)$, there will be no backscattered ray because all creeping rays go to the vertex and diffract in different directions. At $\Psi=270^{\circ}\left(\Psi_{2}=90^{\circ}\right)$, however, there are infinitely many backscattered rays resulting in high observability of the object in this incident direction, and therefore the values are not shown. Because of the excision, the longest backscattered ray (illustrated by $\times$ ) can be captured only for $\Psi \in\left[220^{\circ}, 320^{\circ}\right]\left(\Psi_{2} \geq 40^{\circ}\right)$. Bottom figure shows the total amplitude, $A_{\text {tot }}=\sqrt{A_{1}^{2}+A_{2}^{2}+A_{3}^{2}+A_{4}^{2}}$, of all four backscattered creeping rays.

which is a Hamilton-Jacobi equation. The Hamiltonian for the eikonal equation can be written in the form

$$
H(\mathbf{x}, \mathbf{p})=c(\mathbf{x})|\mathbf{p}| \equiv 1
$$

where $c(\mathbf{x})=1 / n(\mathbf{x})$ is the wave speed and $\mathbf{p}=\nabla \phi$. Introducing the arc length parameter $\tau$, a ray trajectory $(\mathbf{u}(\tau), \mathbf{p}(\tau))$ in $\Omega \times \mathbb{R}^{2}$ is given by the Hamiltonian system 


$$
\begin{aligned}
& \dot{\mathbf{x}}=c(\mathbf{x}) \frac{\mathbf{p}}{|\mathbf{p}|}=c^{2}(\mathbf{x}) \mathbf{p}, \\
& \dot{\mathbf{p}}=-|\mathbf{p}| \nabla_{\mathbf{x}} c(\mathbf{x})=-\frac{\nabla_{\mathbf{x}} c(\mathbf{x})}{c(\mathbf{x})}
\end{aligned}
$$

where the dot denotes differentiation with respect $\tau$. Since $\dot{\mathbf{x}}=J(\mathbf{u}) \dot{\mathbf{u}}$ with the Jacobian $J=\left[\bar{X}_{u} \bar{X}_{v}\right] \in \mathbb{R}^{2 \times 2}$, we have

$$
\dot{\mathbf{u}}=J^{-1}(\mathbf{u}) \dot{\mathbf{x}}=c^{2}(\bar{X}(\mathbf{u})) J^{-1}(\mathbf{u}) \mathbf{p} .
$$

Moreover, inspired by $|\mathbf{p}|=\frac{1}{c(\mathbf{x})}$, we set $\mathbf{p}=\left(p_{1}, p_{2}\right)^{\top}=\frac{1}{c(\mathbf{x})}(\cos \theta, \sin \theta)^{\top}$. Differentiating $\mathbf{p}$ with respect to $\tau$, we get

$$
\dot{\mathbf{p}}=\left(\begin{array}{l}
\nabla_{\mathbf{x}} \frac{1}{c(\mathbf{x})} \cdot \dot{\mathbf{x}} \cos \theta-\frac{1}{c(\mathbf{x})} \dot{\theta} \sin \theta \\
\nabla_{\mathbf{x}} \frac{1}{c(\mathbf{x})} \cdot \dot{\mathbf{x}} \sin \theta+\frac{1}{c(\mathbf{x})} \dot{\theta} \cos \theta
\end{array}\right) .
$$

By (6.3) and (6.5), we get $\dot{\theta}=c_{x}(\mathbf{x}) \sin \theta-c_{y}(\mathbf{x}) \cos \theta$. Therefore, setting $\gamma:=(u, v, \theta) \in$ $\Omega_{p}$, the function $\mathbf{g}(\gamma)$ in $(2.1)$ will be

$$
\mathbf{g}(\gamma)=\left(\begin{array}{c}
c(\bar{X}(\mathbf{u}))\left(g^{11} \cos \theta+g^{12} \sin \theta\right) \\
c(\bar{X}(\mathbf{u}))\left(g^{21} \cos \theta+g^{22} \sin \theta\right) \\
c_{x}(\bar{X}(\mathbf{u})) \sin \theta-c_{y}(\bar{X}(\mathbf{u})) \cos \theta
\end{array}\right)
$$

where $\left(g^{i j}\right)=J^{-1}(\mathbf{u})$. Note that since $\dot{\mathbf{x}} \| \mathbf{p}$ by $(6.3)$, the angle $\theta$ represents the direction of the ray trajectory at $\mathbf{x}$ in the physical space. Moreover, with our choice of Hamiltonian,

$$
\dot{\phi}(\mathbf{x}(\tau))=\nabla \phi(\mathbf{x}(\tau)) \cdot \dot{\mathbf{x}}(\tau)=\mathbf{p} \cdot \mathbf{p} \frac{c(\mathbf{x}(\tau))}{|\mathbf{p}|}=|\mathbf{p}| c(\mathbf{x}(\tau))=1
$$

implying that $\phi$ corresponds to travel-time.

6.2. Multiple-patch scheme. We assume that the physical domain, representing a medium, is a two-dimensional compact manifold $M \subset \mathbb{R}^{2}$ with boundary. Since the wave speed distribution in a multi-layered inhomogeneous medium is not continuous, it is natural to split the medium into different patches with continuous wave speed distributions. We now let $M$ be described by an atlas of charts $\left(M_{j}, w_{j}\right)$ as before. The three-dimensional unit tangent bundle $U T M$ is embedded in $\mathbb{R}^{4}$. In this case, there is an easier way to represent UTM by simplifying the mapping $W_{j}: U T M_{j} \rightarrow \Omega_{p}$ to be $W_{j}(\Gamma)=\gamma$, where

$$
\Gamma=(\mathbf{x}, \mathbf{q}), \quad \mathbf{q}=\hat{s}(\theta)=\left(\begin{array}{c}
\cos \theta \\
\sin \theta
\end{array}\right), \quad \gamma=\left(w_{j}(\mathbf{x}), \theta\right) .
$$

In the same way as before, we can define and compute multiple-patch escape functions $\mathbf{F}(\Gamma)$ and $\boldsymbol{\Phi}(\Gamma)$. However, here the rays are not continuous at the patch boundaries due to the change of the wave speed at these points. When a ray passes the boundary between two layers (two neighboring patches) with different wave speeds, part of the ray is reflected (by the law of reflection), and part of it is refracted or transmitted into the second layer (by Snell's law of refraction). At each interface, therefore, the ray 
field splits into two new ray families, one reflected and one transmitted. Figure 6.1 a shows the reflection and refraction of a ray at the interface between two media of different wave speeds, with $c_{L}>c_{R}$. The law of reflection gives the relation between the angles of incidence $\left(\theta_{\text {inc }}\right)$ and of reflection $\left(\theta_{\text {ref }}\right)$ as

$$
\theta_{\text {inc }}=\theta_{\text {ref }}
$$

The relation between the angles of incidence and of refraction $\left(\theta_{\mathrm{tr}}\right)$ for a ray crossing a boundary between different media is given by Snell's law

$$
\frac{\sin \theta_{i n c}}{\sin \theta_{t r}}=\frac{c_{L}}{c_{R}} .
$$

When a ray moves from a dense to a less dense medium $\left(c_{L}<c_{R}\right)$, Snell's law cannot be used to calculate the refracted angle if $\sin \theta_{\mathrm{tr}}=\sin \theta_{\mathrm{inc}}\left(c_{R} / c_{L}\right)>1$. At this point, the ray is reflected in the incident medium, known as internal reflection. There is therefore a critical angle $\left(\theta_{\mathrm{cr}}\right)$ for which the ray travels directly along the surface between the two refractive media. The critical angle is found by Snell's law, putting in a transmitted angle of 90 degrees. This gives:

$$
\theta_{c r}=\arcsin \frac{c_{R}}{c_{L}}
$$

For any angle of incidence larger than the critical angle $\left(\theta_{\text {inc }}>\theta_{\text {cr }}\right)$, the ray is totally reflected off the interface, obeying the law of reflection. This phenomenon is called total internal reflection. See Figure 6.1b. From (6.8)-(6.10), we can easily find the

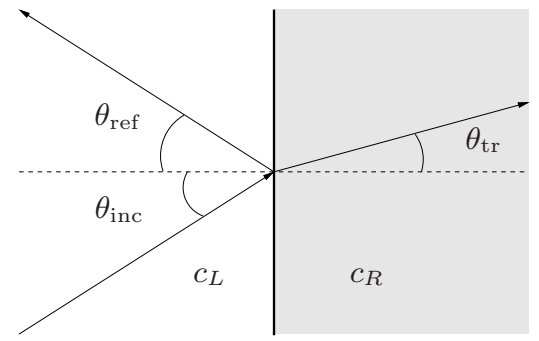

(a)

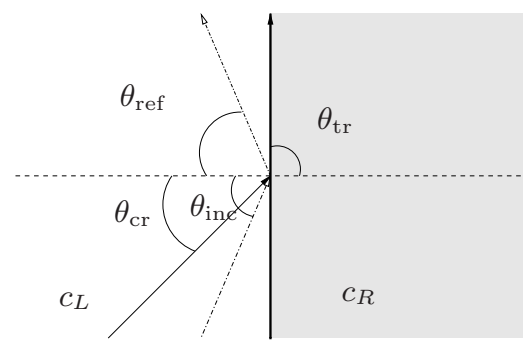

(b)

FIG. 6.1. Reflection and refraction of a ray at the interface between two media of different wave speeds. The left figure shows the reflection and refraction when $c_{L}>c_{R}$. The right figure shows the internal reflection when $\theta_{\mathrm{inc}} \geq \theta_{\mathrm{cr}}$.

inter-patch boundary functions $\tilde{\mathcal{S}}$ and $\tilde{\mathcal{R}}$ discussed in Section 2.2.1. Post-processing in this case is similar to that of the single-patch case, because the escape boundary that we chose coincides with the external boundary of the medium. Note that the inter-patch boundary conditions above can be seen as a way to preserve the Hamiltonian (6.2) for a ray across the patch boundary. In cases where the discontinuity in $c(\mathbf{x})$ is not aligned with the patch boundary, the solution of the escape equations is not unique. Uniqueness can, however, be recovered by enforcing the extra condition that solutions should be continuous along constant Hamiltonian paths also inside the patches. This is the idea of so called Hamiltonian-preserving methods developed in $[14,15]$. These methods capture the effect of a discontinuous $c(\mathbf{x})$ on uniform grids not aligned with the discontinuity. 
6.3. Example 3-a multi-layered medium. We consider a multi-layered medium $M=[0,6]^{2}$ consisting of three layers with different wave speeds (see Figure 6.2):

- Top layer:

$$
\begin{aligned}
c_{1}(x, y) & =1+0.05(x-3)^{2}+0.25 y, \\
c_{2}(x, y) & =\frac{3}{1+e^{-\left((x-3)^{2}+(y-3)^{2}\right)}},
\end{aligned}
$$

- Middle layer:

- Bottom layer: $\quad c_{3}(x, y)=0.5+0.2 x+0.5 y$.

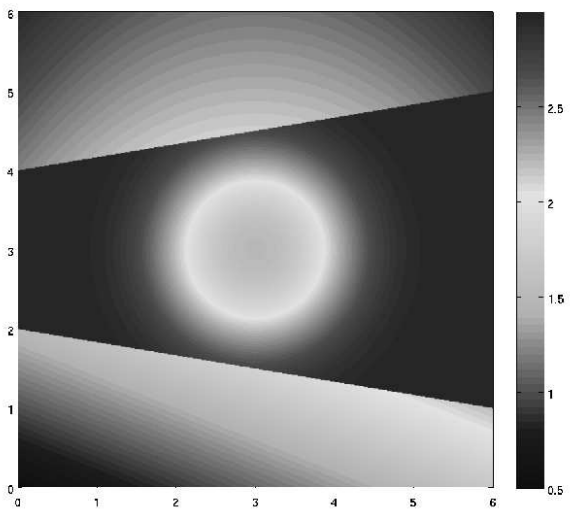

FIG. 6.2. The medium consisting of three layers and grey scale plot of the wave speed field.

We want to compute multi-valued travel-time of seismic rays in the medium from a given source point $\mathbf{x}_{0}$ on the boundary. We split the medium into three patches corresponding to the three layers, as shown in Figure 6.2. The escape equations for the escape point $F$ and the travel-time $\Phi$ are derived and solved in each patch. In order to find the travel-time with a given source point by post-processing, we first choose the four outermost boundaries of the entire physical domain as the escape boundary. We then continue as follows:

0 . The source point $\mathbf{x}_{0}$ on the boundary is first reduced to a point $S_{0} \in \mathbb{R}$.

1. For each point $\mathbf{x} \in M$, find $\mathbf{F}(\Gamma)=(\mathbf{U}, \mathbf{V}, \boldsymbol{\Theta})$ with $\Gamma=(\mathbf{x}, \mathbf{q}(\theta))$ for all $\theta \in \mathbb{S}$. Now $(\mathbf{U}, \mathbf{V})$ can again be reduced to points $S \in \mathbb{R}$, parameterized by $\theta$.

2. Find $\theta=\theta^{*}$ such that $S_{0}=S(\theta)$.

3. Travel-time at $\mathbf{x} \in M$ will then be $\mathbf{\Phi}\left(\Gamma^{*}\right)$ with $\Gamma^{*}=\left(\mathbf{x}, \mathbf{q}\left(\theta^{*}\right)\right)$.

Figure 6.3 shows the distribution of transmitted seismic rays and equi-arrival curves, i.e., the locus of all points in physical domain which have the same travel-time, from two different source points, $\mathbf{x}_{0}=(3,6)$ and $\mathbf{x}_{0}=(3.5,6)$. Note that we can track both reflected and transmitted ray families, but not at the same time. In order to get all rays, one needs to follow all ray families. Figure 6.4 shows the equi-arrival curves of rays reflected from the top and bottom interfaces inside the top and the middle layers, respectively, for a source point at $\mathbf{x}_{0}=(3,6)$. If we repeat this procedure, we can also capture multiple rays reflected from the two interfaces that get trapped inside the middle layer and reverberate to infinity. Here, we do not consider reflections from the domain boundaries, as we have a truncated domain much smaller than the physical space in which the waves propagate. 

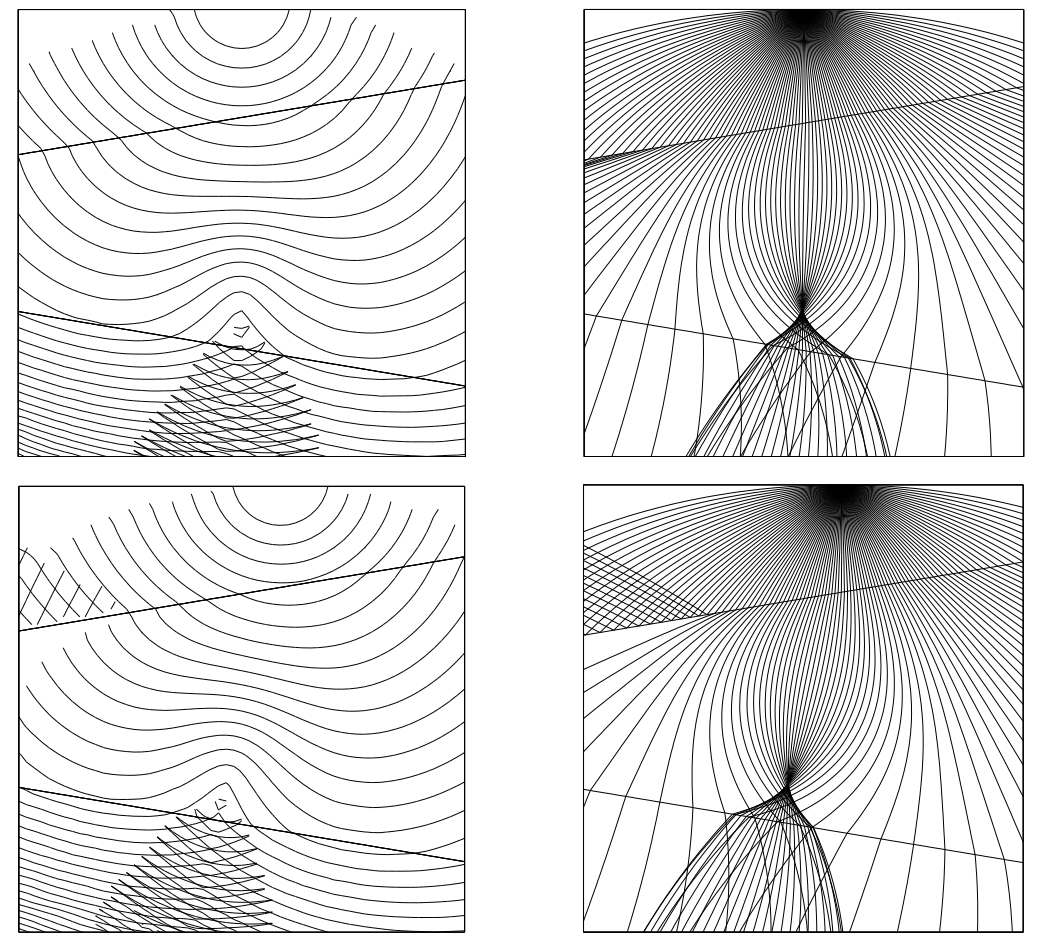

FIG. 6.3. The equi-arrival curves and the distribution of seismic rays for two different source points.
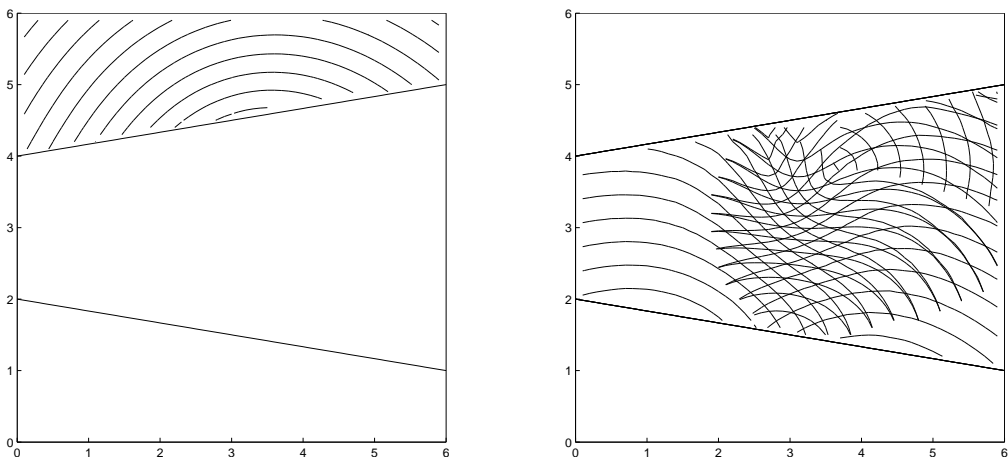

FIG. 6.4. The equi-arrival curves of reflected seismic rays from the top (left figure) and bottom (right figure) interfaces for a source point on the center of the top of the domain.

\section{Conclusion}

We have modified the single-patch phase space method for computing creeping rays to a multiple-patch method for computing trajectories on two-dimensional manifolds possibly embedded in a higher-dimensional space. The dynamics of trajectories are given by systems of first-order ODEs in a phase space. We split the manifold into multiple patches where each patch has a well-defined regular parameterization. The escape equations, which are hyperbolic PDEs in a three-dimensional phase space, are 
derived and solved in each patch, individually, using a second-order version of the fast marching method. The solutions of individual patches are then connected using suitable inter-patch boundary conditions. Properties for particular families of trajectories are obtained through a fast post-processing. For some applications, the complexity of the method is attractive. Such applications include mono-static and bi-static RCS computations, antenna coupling problems, and travel-time computations of seismic waves when the solution is sought for many different sources.

\section{REFERENCES}

[1] N.C. Albertsen, Creeping wave modes for a dielectric coated cylinder, IEEE T. Antenn. Propag., 37(12), 1642-1644, 1989.

[2] C. Belta and V. Kumar, Optimal motion generation for groups of robots: A geometric approach, J. Mech. Des., 126, 36-70, 2004.

[3] D.P. Bouche, J.J. Bouquet, H. Manenc, and R. Mittra, Asymptotic computation of the RCS of low observable axisymmetric objects at high frequency, IEEE T. Antenn. Propag., 40(10), 1165-1174, 1992.

[4] H. Brandén and S. Holmgren, Convergence acceleration for hyperbolic systems using semicirculant approximations, J. Sci. Comput., 14(4), 357-393, 1999.

[5] T.F. Chan and T.P. Mathew, Domain decomposition algorithms, Acta Numerica, 3, 61-143, 1994.

[6] V. Cervený, I.A. Molotkov and I. Psencik, Ray Methods in Seismology, Univ. Karlova Press, 1977.

[7] B. Engquist and O. Runborg, Computational high frequency wave propagation, Acta Numerica, $12,181-266,2003$.

[8] E. Fatemi, B. Engquist and S.J. Osher, Numerical solution of the high frequency asymptotic expansion for the scalar wave equation, J. Comput. Phys., 120(1), 145-155, 1995.

[9] S. Fomel and J.A. Sethian, Fast phase space computation of multiple arrivals, Proc. Natl. Acad. Sci. USA, 99(11), 7329-7334 (electronic), 2002.

[10] S. Hagdahl, Hybrid Methods for Computational Electromagnetics in Frequency Domain, PhD thesis, NADA, KTH, Stockholm, 2005.

[11] M. Hilaga, Y. Shinagawa, T. Kohmura and T. L. Kunii, Topology matching for fully automatic similarity estimation of $3 D$ shapes, in Proceedings of the 28th annual conference on Computer graphics and interactive techniques, 203-212, 2001. ACM Press, New York, NY, USA.

[12] S. Holmgren and K. Otto, Semicirculant preconditioners for first-order partial differential equations, SIAM J. Sci. Comput., 15, 385-407, 1994.

[13] P.E. Hussar, V. Oliker, H.L. Riggins, E.M. Smith-Rowlan, W.R. Klocko and L. Prussner, An implementation of the UTD on facetized CAD platform models, IEEE Antennas Propag., 42(2), 100-106, 2000.

[14] S. Jin and X. Wen, Hamiltonian-preserving schemes for the Liouville equation with discontinuous potentials, Commun. Math. Sci., 3, 285-315, 2005.

[15] S. Jin and X. Wen, Hamiltonian-preserving schemes for the Liouville equation of geometrical optics with discontinuous local wave speeds, J. Comput. Phys., 214(2), 672-697, 2006.

[16] K.H. Karlsen, K.A. Lie and N.H. Risebro, A fast marching method for reservoir simulation, Computational Geosciences, 4(2), 185-206, 2000.

[17] J. Keller and R.M. Lewis, Asymptotic methods for partial differential equations: the reduced wave equation and Maxwell's equations, Surveys Appl. Math., 1, 1-82, 1995.

[18] J.B. Keller, The geometric theory of diffraction, in Symposium on Microwave Optics, Eaton Electronics Research Laboratory, McGill University, Montreal, Canada, June, 1953.

[19] L.C. Kempel, J.L. Volakis and S. Bindiganavale, Radiation and scattering from printed antennas on cylindrically conformal platforms, Technical report, Michigan Univ. Final Report, January, 1994

[20] R. Kimmel and J.A. Sethian, Computing geodesic paths on manifolds, Proc. Natl. Acad. Sci. USA, 95(15), 8431-8435 (electronic), 1998.

[21] E.M. Koper, W.D. Wood and S.W. Schneider, Aircraft antenna coupling minimization using genetic algorithms and approximations, IEEE T. Aero. Elec. Sys., 40(2), 742-751, 2004

[22] A.P. Krasnojen, Features of creeping waves propagation on the dielectric-coated circular cylinder, IEE Proc. Microw. Antennas Propag., 145(2), 179-183, 1998.

[23] K.M. Kuperberg and C.S. Reed, A dynamical system on $\mathbb{R}^{3}$ with uniformly bounded trajectories 
and no compact trajectories, in Proceedings of the American Mathematical Society, 106, 1095-1097, 1989.

[24] H. Ling, R. Chou and S. W. Lee, Shooting and bouncing rays: Calculating the RCS of an arbitrarily shaped cavity, IEEE T. Antenn. Propag., 37, 194-205, 1989.

[25] H. Liu, S. Osher and R. Tsai, Multi-valued solution and level set methods in computational high frequency wave propagation, Commun. Comput. Phys., 1, 765-804, 2006.

[26] M. Motamed and O. Runborg, A fast phase space method for computing creeping rays, J. Comput. Phys., 219(1), 276-295, 2006.

[27] R. Paknys and D.R. Jackson, The relation between creeping waves, leaky waves, and surface waves, IEEE T. Antenn. Propag., 53(3), 898-907, 2005.

[28] R. Paknys and N. Wang, Creeping wave propagation constants and modal impedence for a dielectric coated cylinder, IEEE T. Antenn. Propag., 34(5), 674-680, 1986.

[29] J. Perez, J.A. Saiz, O.M. Conde, R.P. Torre and M.F. Catedra, Analysis of antennas on board arbitrary structures modeled by NURBS surfaces, IEEE T. Antenn. Propag., 45(6), 1045$1053,1997$.

[30] J. Qian and W.W. Symes, An adaptive finite-difference method for traveltimes and amplitudes, Geophysics, 67(1), 167-176, January-February, 2002.

[31] O. Runborg, Mathematical models and numerical methods for high frequency waves, Commun. Comput. Phys., 2, 827-880, 2007.

[32] J. Shim and H.T. Kim, Dominance of creeping wave modes of backscattered field from a conducting sphere with dielectric coating, Progress In Electromagnetic Research, 21, 293-306, 1999.

[33] Chi-Wang Shu, Essentially non-oscillatory and weighted essentially non-oscillatory schemes for hyperbolic conservation laws, ICASE Report 97-65, Brown University, 1997. Prepared for NASA Langley Research Center.

[34] W.W. Symes and J. Qian, A slowness matching Eulerian method for multivalued solutions of eikonal equations, J. Sci. Comput., 19(1-3), 501-526, 2003.

[35] G. Taylor, Another look at the line intersection problem, Int. J. Geogr. Inf. Syst., 3(20), 192-193, 1989.

[36] J. van Trier and W.W. Symes, Upwind finite-difference calculation of traveltimes, Geophysics, 56(6), 812-821, June, 1991.

[37] J. Vidale, Finite-difference calculation of traveltimes, B. Seismol. Soc. Am., 78(6), 2062-2076, December, 1988.

[38] V. Vinje, E. Iversen and H. Gjøystdal, Traveltime and amplitude estimation using wavefront construction, Geophysics, 58(8), 1157-1166, 1993.

[39] L. Ying and E.J. Candes, Fast geodesics computation with the phase flow method, J. Comput. Phys., 220(1), 6-18, 2006. 